\title{
Kisspeptin receptor agonist has therapeutic potential for female reproductive disorders
}

\author{
Ali Abbara, ${ }^{1}$ Pei Chia Eng, ${ }^{1}$ Maria Phylactou, ${ }^{1}$ Sophie A. Clarke, ${ }^{1}$ Rachel Richardson, ${ }^{2}$ Charlene M. Sykes, ${ }^{3}$ Chayarndorn Phumsatitpong, ${ }^{3}$ \\ Edouard Mills, ${ }^{1}$ Manish Modi, ${ }^{1}$ Chioma Izzi-Engbeaya, ${ }^{1}$ Debbie Papadopoulou, ${ }^{1}$ Kate Purugganan, ${ }^{4}$ Channa N. Jayasena, ${ }^{1}$ \\ Lisa Webber, ${ }^{5}$ Rehan Salim, ${ }^{4}$ Bryn Owen, ${ }^{1}$ Paul Bech, ${ }^{1}$ Alexander N. Comninos, ${ }^{1}$ Craig A. McArdle, ${ }^{6}$ Margaritis Voliotis, ${ }^{7}$ \\ Krasimira Tsaneva-Atanasova, ${ }^{7,8}$ Suzanne Moenter, ${ }^{3,9,10}$ Aylin Hanyaloglu, ${ }^{2}$ and Waljit S. Dhillo ${ }^{1}$ \\ 'Section of Endocrinology and Investigative Medicine, Imperial College London, Hammersmith Hospital, London, United Kingdom. ${ }^{2}$ Institute of Reproductive and Developmental Biology, Department \\ of Metabolism, Digestion and Reproduction, Imperial College London, London, United Kingdom. ${ }^{3}$ Department of Molecular \& Integrative Physiology, University of Michigan, Ann Arbor, Michigan, USA. \\ ${ }^{4}$ Hammersmith IVF unit, and ${ }^{5}$ St Mary's Hospital, Imperial College Healthcare NHS Trust, London, United Kingdom. ${ }^{6}$ Department of Translational Medicine, Bristol Medical School, University of Bristol, Bristol, \\ United Kingdom. 'Department of Mathematics and Living Systems Institute, and ${ }^{8}$ EPSRC Centre for Predictive Modelling in Healthcare, University of Exeter, Exeter, United Kingdom. ${ }^{9}$ Department of Internal \\ Medicine, and ${ }^{10}$ Department of Obstetrics and Gynecology, University of Michigan, Ann Arbor, Michigan, USA.
}

\begin{abstract}
BACKGROUND. Kisspeptin is a key regulator of hypothalamic gonadotropin-releasing hormone (GnRH) neurons and is essential for reproductive health. A specific kisspeptin receptor (KISS1R) agonist could significantly expand the potential clinical utility of therapeutics targeting the kisspeptin pathway. Herein, we investigate the effects of a KISS1R agonist, MVT602, in healthy women and in women with reproductive disorders.
\end{abstract}

METHODS. We conducted in vivo and in vitro studies to characterize the action of MVT-602 in comparison with native kisspeptin-54 (KP54). We determined the pharmacokinetic and pharmacodynamic properties of MVT-602 (doses 0.01 and $0.03 \mathrm{nmol} / \mathrm{kg}$ ) versus KP54 $(9.6 \mathrm{nmol} / \mathrm{kg})$ in the follicular phase of healthy women $(n=9)$, and in women with polycystic ovary syndrome (PCOS; $n=6$ ) or hypothalamic amenorrhea (HA; $n=6)$. Further, we investigated their effects on KISS1Rmediated inositol monophosphate (IP) and $\mathrm{Ca}^{2+}$ signaling in cell lines and on action potential firing of $\mathrm{GnRH}$ neurons in brain slices.

RESULTS. In healthy women, the amplitude of luteinizing hormone (LH) rise was similar to that after KP54, but peaked later (21.4 vs. 4.7 hours; $P=0.0002$ ), with correspondingly increased AUC of LH exposure (169.0 vs. $38.5 \mathrm{IU} \cdot \mathrm{h} / \mathrm{L} ; \boldsymbol{P}=0.0058$ ). LH increases following MVT-602 were similar in PCOS and healthy women, but advanced in HA $(P=0.004)$. In keeping with the clinical data, MVT-602 induced more potent signaling of KISS1R-mediated IP $P_{1}$ accumulation and a longer duration of GnRH neuron firing than KP54 (115 vs. 55 minutes; $P=0.0012$ ).

CONCLUSION. Taken together, these clinical and mechanistic data identify MVT-602 as having considerable therapeutic potential for the treatment of female reproductive disorders.

TRIAL REGISTRATION. International Standard Randomised Controlled Trial Number (ISRCTN) Registry, ISRCTN21681316.

FUNDING. National Institute for Health Research and NIH.

\section{Introduction}

Kisspeptin is recognized to play a pivotal role in the regulation of reproductive hormone secretion following 2 landmark reports in 2003, demonstrating that decreased signaling of its G proteincoupled receptor (KISS1R) resulted in failure of the hypothalamicpituitary-gonadal (HPG) axis $(1,2)$. Thereafter, it was estab-

Authorship note: $A A, P C E$, and MP are co-first authors.

Conflict of interest: AA and WSD have conducted consulting work for Myovant Sciences Ltd.

Copyright: (c) 2020, American Society for Clinical Investigation.

Submitted: May 15, 2020; Accepted: September 3, 2020; Published: November 16, 2020.

Reference information: J Clin Invest. 2020;130(12):6739-6753.

https://doi.org/10.1172/JCl139681. lished that kisspeptin activates gonadotropin-releasing hormone $(\mathrm{GnRH})$ neurons in the hypothalamus, thereby stimulating the downstream HPG axis $(3,4)$. Current evidence suggests that kisspeptin neurons are key integrators of peripheral signals including sex steroids and metabolic cues $(5,6)$.

The kisspeptins are a family of peptides encoded by the KISS1 gene (7); the 145-amino acid precursor protein is proteolytically cleaved to shorter peptides, and the number of remaining amino acids is indicated by their suffix, e.g., KP54. Exogenous kisspeptin-54 (KP54) administration robustly stimulates gonadotropin secretion in both healthy men $(8)$ and women $(9,10)$. Due to its fundamental role in regulating physiological reproductive hormone secretion, there has been tremendous interest in targeting the kisspeptin pathway to treat reproductive disorders in humans 
Table 1. Baseline characteristics of healthy women, women with HA, and women with PCOS

$\begin{array}{lcccc}\text { Clinical characteristics } & \text { Healthy women }(\boldsymbol{n}=\mathbf{9}) & \text { Women with PCOS }(\boldsymbol{n}=\mathbf{6}) & \text { Women with HA }(\boldsymbol{n}=\mathbf{6}) & \boldsymbol{P} \text { value } \\ \text { Age (years) } & 26.0(21.0,32.8) & 24.5(21.0,26.3) & 25.0(23.0,30.8) & 0.56 \\ \text { Mass (kg) } & 64.7(43.4,72.6) & 61.2(51.9,72.1) & 55.3(51.5,61.0) & 0.28 \\ \text { BMI (kg/m²) } & 24.5(20.1,25.5) & 23.2(18.4,25.1) & 20.7(19.4,23.1) & 0.11 \\ \text { Serum LH (IU/L) } & 3.7(3.0,4.4) & 4.4(1.9,9.3) & 2.9(1.4,3.6) & 0.22 \\ \text { Serum FSH (IU/L) } & 5.1(2.9,5.9) & 4.3(2.9,5.0) & 5.2(4.6,6.3) & 0.22 \\ \text { Serum estradiol (pmol/L) } & 93.0(72.3,135.3) & 81.0(67.3,107) & 72.5(50.5,110.3) & 0.47 \\ \text { SHBG (nmol/L) } & 69.0(48.8,109.5) & 32.0(31.3,48.3) & 65.0(39.8,100.3) & 0.04 \\ \text { Serum AMH (pmol/L) } & 23.9(2.4,36.8) & 70.7(29.8,104.3) & 20.8(14.9,23.2) & 0.01\end{array}$

Median (25th centile, 75th centile) is presented of hormone levels on the morning of the first study visit. Comparison was made using Kruskal-Wallis test with post hoc Dunn's multiple-comparisons test. Women with PCOS had significantly lower sex hormone-binding globulin (SHBC) and higher AMH compared with healthy women and women with HA. LH, luteinizing hormone; FSH, follicle-stimulating hormone; AMH, anti-Müllerian hormone.

(11). It has been shown that a single subcutaneous (s.c.) bolus of KP54 induces a luteinizing hormone (LH) rise that safely matures oocytes in women undergoing in vitro fertilization (IVF) therapy $(12,13)$. Additionally, KP54 administration can restore physiological reproductive hormone secretion in patients with functional hypogonadism associated with deficient GnRH secretion such as in hypothalamic amenorrhea (HA) (14). However, therapeutic use of native KP54 using frequent high-dose administration risks tachyphylaxis and resultant insufficient stimulation of the reproductive axis $(15,16)$. Consequently, the development of KISS1R agonists with a longer duration of action could allow for less frequent dosing than native KP54 and could capitalize on the potential clinical utility of kisspeptin-based therapeutics.

All kisspeptin peptides share a common C-terminal decapeptide sequence, equivalent to KP10, and activate the $\mathrm{G} \alpha_{\mathrm{q}} / 11$-coupled KISS1R, leading to stimulation of phospholipase $C$ and increases in the intracellular second messengers diacylglycerol and inositol 1,4,5-trisphosphate ( $\left.\mathrm{IP}_{3}\right)$, and release of $\mathrm{Ca}^{2+}$ from intracellular stores (17). KP54 is the major circulating form of kisspeptin in humans and has a terminal half-life of 27.6 minutes (8). Kisspeptin-10 (KP10) has also been widely studied in humans (10, $18)$, but has a shorter terminal half-life of 4 minutes $(8,19)$.

Recently, KISS1R agonists have been developed through modification of KP10 to have increased potency and stability in order to advance kisspeptin-targeted therapeutics through the translational pathway (20-22). MVT-602 (previously known as TAK-448) is a KISS1R agonist with a longer duration of action than native KP54 (8, 23). In healthy men, a single dose of MVT-602 induced sustained stimulation with peak gonadotropin levels occurring between 6 and 12 hours and levels remaining elevated for 48-72 hours (23).

MVT-602 is expected to also have substantial translational potential for the treatment of female reproductive disorders, but its effects in women have not been investigated. We therefore aimed to comprehensively determine the pharmacokinetic (PK) and pharmacodynamic (PD) profiles of the KISS1R agonist MVT-602 in healthy women and in the 2 most common forms of oligo/anovulatory subfertility, polycystic ovary syndrome (PCOS) and HA (24-26). Additionally, we investigated the effects of MVT-602 and KP54 on intracellular signaling following activation of the $G$ protein-coupled KISS1R, and on action potential firing of GnRH neurons in brain slices. Furthermore, as the sex steroid milieu at the time of administration is known to influence the response to kisspeptin (9), we also sought to determine the impact of estrogen supplementation on the gonadotropin response to MVT-602 in women.

\section{Results}

Baseline characteristics of participants are summarized in Table 1. Age, body mass, and body mass index (BMI) did not differ between healthy women, women with PCOS, and women with HA. All healthy women had regular menstrual cycles with an average cycle length of $28.1 \pm 1.2$ days, whereas all women with PCOS or HA were oligo/amenorrheic. Women with PCOS had higher serum anti-Müllerian hormone levels and lower sex hormonebinding globulin (SHBG) levels as expected. No serious adverse effects were identified after injection of either MVT-602 or KP54.

Preliminary dose-finding study of MVT-602 in healthy women. As MVT-602 had not been previously administered to women, a broad range of doses between $0.003 \mathrm{nmol} / \mathrm{kg}$ and $1.0 \mathrm{nmol} / \mathrm{kg}$ of MVT-602 administered as a single s.c. bolus were evaluated in the early follicular phase (days 1-4 of the menstrual cycle) of healthy women $(n=3)$ during a preliminary dose-finding study. The highest LH levels occurred 24 hours after administration, and no greater increases in serum gonadotropins or estradiol levels were observed with doses higher than $0.03 \mathrm{nmol} / \mathrm{kg}$. Therefore, detailed endocrine profiles were evaluated in subsequent phases of the study using MVT-602 doses of 0.01 and $0.03 \mathrm{nmol} / \mathrm{kg}$ (Figure 1).

Comparison of MVT-602 with KP54 in the follicular phase of healthy women. We compared MVT-602 with a dose of KP54 (9.6 $\mathrm{nmol} / \mathrm{kg}$ ) that is known to induce a near-maximal response on $\mathrm{LH}$ in women $(9,12,13)$. Plasma MVT-602 and KP54 levels peaked at 0.4-1 hour after both peptides (Figure 2A and Table 2). Peak MVT602 levels were dose-proportionately higher after $0.03 \mathrm{nmol} / \mathrm{kg}$ than after $0.01 \mathrm{nmol} / \mathrm{kg}\left(C_{\max } 29.5 \mathrm{vs.} 8.1 \mathrm{pmol} / \mathrm{L}\right.$; Table 2$)$. Despite PK parameters being similar for both ligands $\left(t_{1 / 2} 1.68-2.02\right.$ hours; Table 2), the time course of LH release was markedly different (Figure 2B). The effect on LH was modeled using a pharmacodynamic biphasic model providing parameters for both the primary episode of LH response commencing within the first 10 hours after administration and, where present, the secondary episode of 
A

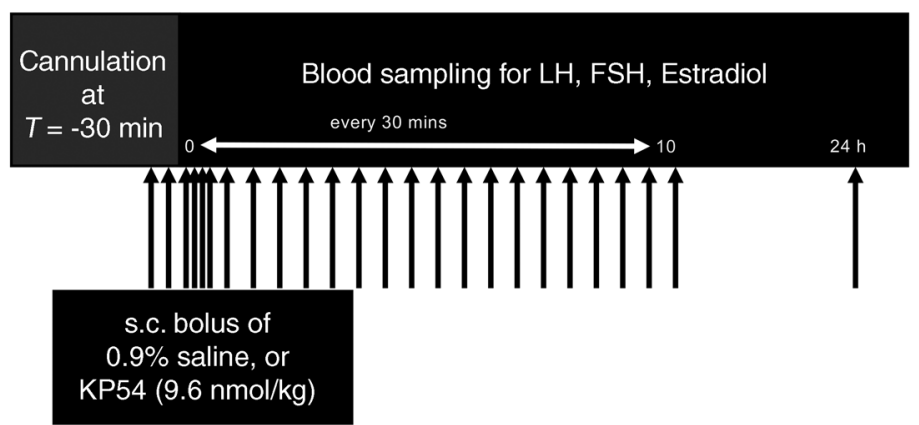

B

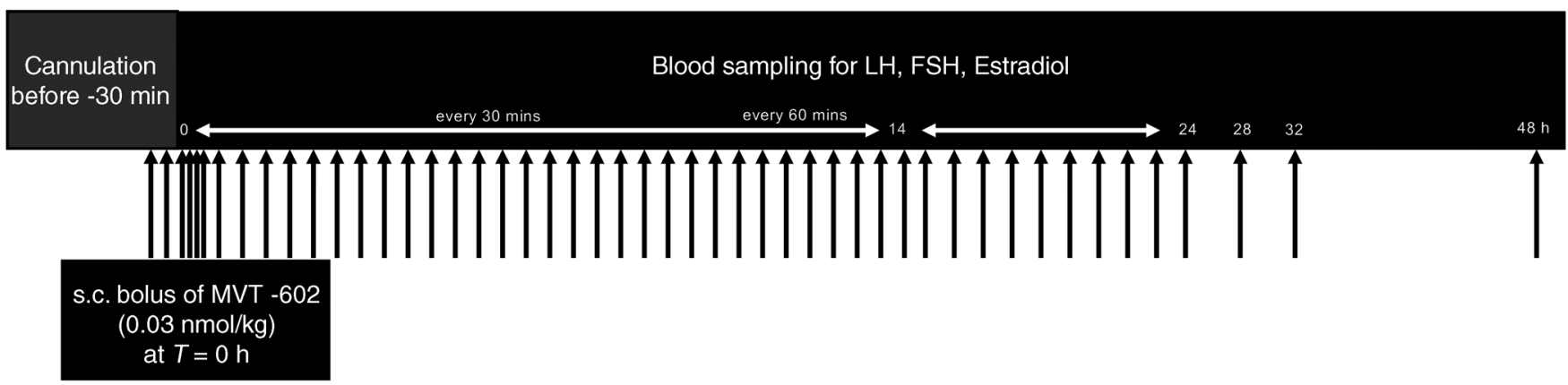

Figure 1. Study protocol. Study participants were admitted to the Clinical Research Facility at 8 am on the morning of each study visit. An i.v. cannula was inserted into one antecubital fossa, and blood was sampled at $T-30$ minutes, $T-15$ minutes, and $T=0$ hours before administration of each intervention to determine the basal hormonal values. An s.c. bolus of kisspeptin-54 (KP54) or $0.9 \%$ saline (A), or of MVT-602 (B), was administered at $T=0$ hours. (A) Study protocol diagram for the KP54 and 0.9\% saline visits. After an s.c. bolus of KP54 ( $9.6 \mathrm{nmol} / \mathrm{kg}$ ) or $0.9 \%$ saline at $T=0$ hours, serum hormone levels (LH, FSH, estradiol, and progesterone) were measured every 5-15 minutes for the first 30 minutes, and then every 30 minutes until 10 hours, and additionally at 24 hours. (B) Study protocol diagram for MVT-602 visits. After an s.c. bolus of MVT-602 $(0.03 \mathrm{nmol} / \mathrm{kg})$ was administered at $T=0$ hours, serum hormone levels ( $\mathrm{LH}, \mathrm{FSH}$, estradiol, and progesterone) were measured every 5-15 minutes for the first 30 minutes, then every 30 minutes until 14 hours, and then every 60 minutes until 24 hours and additionally at 28,32 , and 48 hours. A further blood test at 72 hours was carried out in studies using estradiol pretreatment.

LH response commencing after 10 hours (Table 2). Although the amplitude of LH response was similar after KP54 and MVT-602, the timing of peak LH was much later after MVT-602 than after KP54 (21.4 vs. 4.7 hours; $P=0.0005$ ) (Table 2). Consequently, the area under the curve (AUC) of LH was increased at least 4 -fold after $0.03 \mathrm{nmol} / \mathrm{kg}$ MVT-602 in comparison with KP54 (169.0 vs. $38.5 \mathrm{IU} \cdot \mathrm{h} / \mathrm{L} ; P=0.01$ ) (Figure $2 \mathrm{C}$ and Table 2). Follicle-stimulating hormone (FSH) levels followed a similar trajectory to LH in response to both peptides (Figure 2D). Elevated serum estradiol was maintained for at least 48 hours after a single s.c. injection of 0.01 or $0.03 \mathrm{nmol} / \mathrm{kg}$ MVT-602 (Figure 2E).

Effect of MVT-602 and KP54 on human KISS1R signaling. We next examined whether the prolonged effects of MVT- 602 on serum LH, FSH, and estradiol levels could be explained by differences in signaling at a cellular level. KISS1R signals via the $\mathrm{G} \alpha_{\mathrm{q}} / 11$ pathway (27), leading to stimulation of phospholipase C and increases in the intracellular second messengers diacylglycerol and inositol 1,4,5-trisphosphate $\left(\mathrm{IP}_{3}\right)$, and release of $\mathrm{Ca}^{2+}$ from intracellular stores (17). We thus compared KISS1R-mediated $\mathrm{G} \alpha_{\mathrm{q}} / 11$ signaling following treatment with either KP54 or MVT602. HEK293 cells expressing human KISS1R were treated with peptide, and intracellular levels of inositol monophosphate (IP), a downstream metabolite of $\mathrm{IP}_{3}$, and intracellular $\mathrm{Ca}^{2+}$ were measured (Supplemental Figure 1; supplemental material available online with this article; https://doi.org/10.1172/JCI139681DS1). No signal response was observed from either peptide in cells not transfected with the receptor.

Analysis with varying doses of each ligand $(10 \mathrm{pM}-1 \mu \mathrm{M})$ revealed that the 2 ligands did not significantly differ in efficacy (maximum fluorescence ratio $\left[R_{\max }\right]$ KP54 $=96.05 \pm 3.96$ and MVT- $602=89.14 \pm 6.25$, mean \pm SEM, where data are normalized as a percentage of $1 \mu \mathrm{M}$ KP54). However, MVT-602 was significantly $(P<0.0001)$ more potent (logarithmically transformed $\left.\mathrm{EC}_{50}\left[\mathrm{pEC}_{50}\right] 10.71 \pm 0.12\right)$ than $\mathrm{KP} 54\left(\mathrm{pEC}_{50} 8.04 \pm 0.06\right)$, as evidenced by the leftward-shifted dose-response curve in Figure 3A.

Activation of KISS1R with KP10 induces both a transient and a more sustained intracellular $\mathrm{Ca}^{2+}$ response $(28,29)$. Thus, to determine whether MVT-602 and KP54 resulted in differences in the kinetics of KISS1R-G $\alpha_{q} / 11$ signaling, real-time $\mathrm{Ca}^{2+}$ mobilization was monitored. Cells were treated with $10 \mathrm{nM}$ of either ligand, which is a saturating dose for MVT-602, and a dose of KP54 corresponding to its $\mathrm{EC}_{50}$ in terms of IP levels (Figure 3A). Stimulating cells at higher doses of KP54 did not significantly alter intracellular $\mathrm{Ca}^{2+}$ signal responses (Supplemental Figure 2), suggesting that maximal responses were achieved with $10 \mathrm{nM}$ of either MVT-602 or KP54. Treatment of cells with either KP54 or MVT-602 produced a rapid rise in intracellular $\mathrm{Ca}^{2+}$ (with maximal intensity reached within about $30-60$ seconds) that was sustained over time 
A

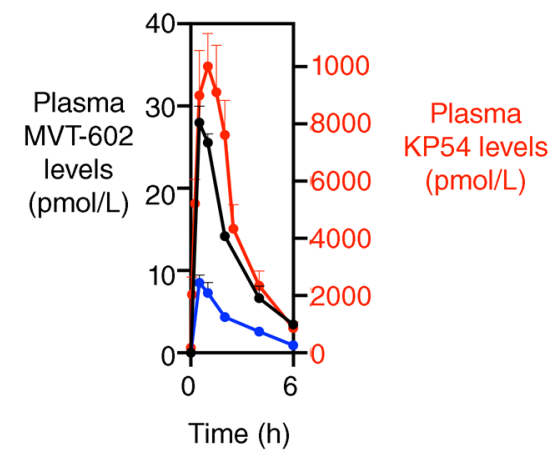

C

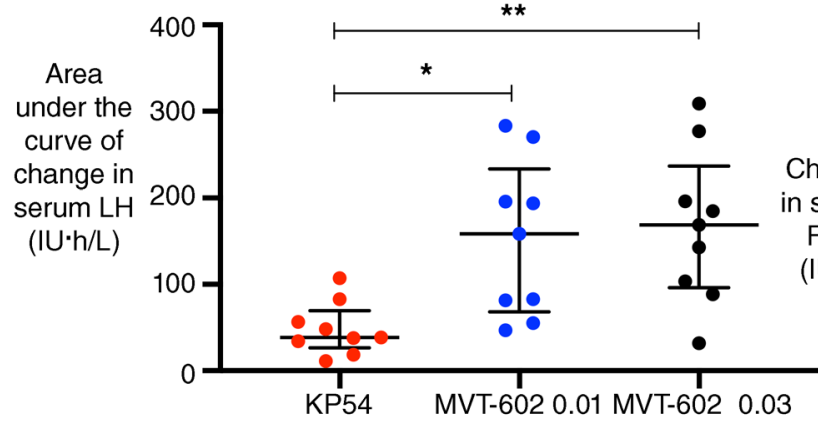

B

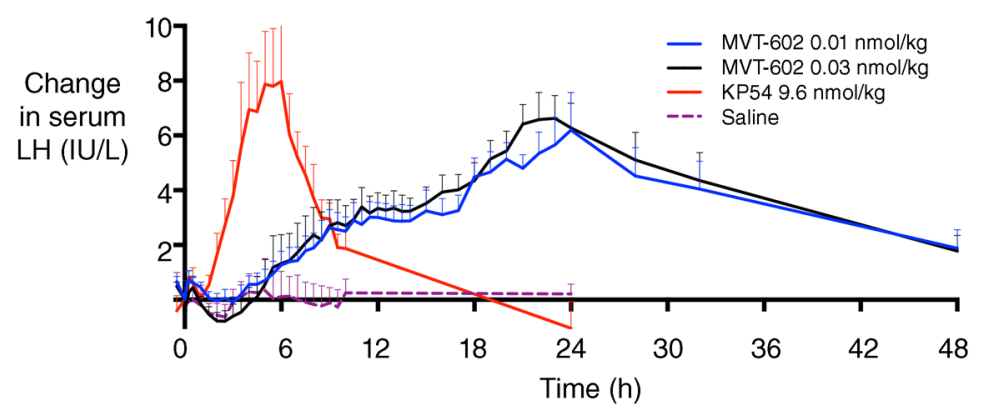

D

E

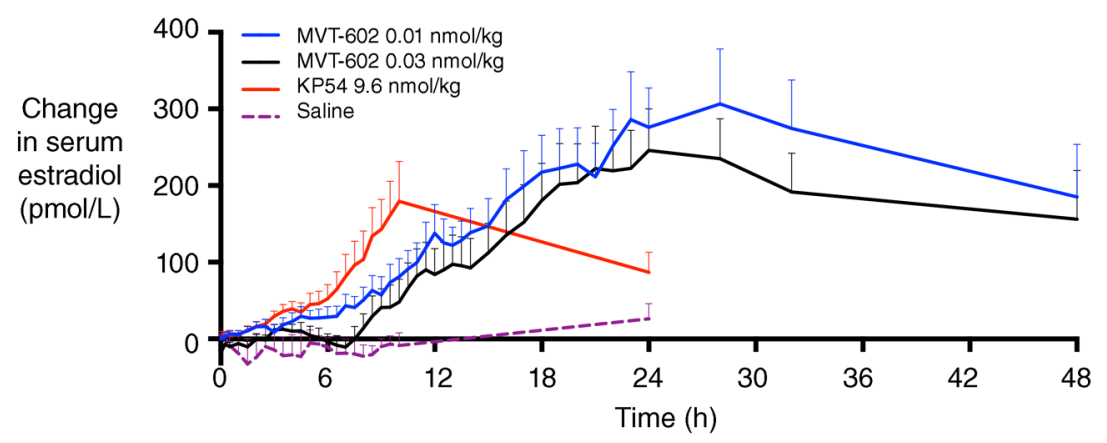

Figure 2. Clinical studies of MVT-602 and KP54 in healthy women in the follicular phase. (A) Mean ( \pm SEM) plasma concentration of MVT-602 (left $y$ axis) or KP54 (right $y$ axis) in pmol/L versus time (hours) in healthy women receiving an s.c. bolus of MVT-602 at $0.03 \mathrm{nmol} / \mathrm{kg}$ (blue) or $0.01 \mathrm{nmol} / \mathrm{kg}$ (black) or of KP54 (red) in the first 6 hours after s.c. administration at time 0 hours of each peptide. (B, D, and E) Mean ( \pm SEM) change from baseline levels in serum LH (IU/L) (B), serum FSH (IU/L) (D), and serum estradiol (pmol/L) (E) in healthy women during the early follicular phase $(n=9)$ receiving an s.c. bolus of MVT-602 at time 0 hours. MVT-602 doses of $0.01 \mathrm{nmol} / \mathrm{kg}$ are presented in blue (over 48 hours), $0.03 \mathrm{nmol} / \mathrm{kg} \mathrm{MVT-602} \mathrm{in} \mathrm{black} \mathrm{(over} 48 \mathrm{hours),} 9.6 \mathrm{nmol} / \mathrm{kg} \mathrm{KP} 54 \mathrm{in}$ red (over 24 hours), and $0.9 \%$ saline in purple (over 24 hours). (C) Median (IQR) of modeled values of AUC of serum LH (IU.h/L) after KP54 (9.6 nmol/kg), MVT$602(0.01 \mathrm{nmol} / \mathrm{kg})$, and MVT-602 $(0.03 \mathrm{nmol} / \mathrm{kg})$. Groups were compared by Kruskal-Wallis test with post hoc Dunn's multiple comparison. The duration of sampling was 24 hours for KP54, as LH had returned to baseline within this time frame, whereas it was 48 hours for MVT-602. ${ }^{*} P<0.05,{ }^{* *} P<0.01$.

(Figure 3B and Supplemental Videos 1 and 2), similar to $\mathrm{Ca}^{2+}$ signal profiles reported for $\operatorname{KP} 10(28,29)$. The acute maximal response was not different following MVT-602 treatment in comparison with KP54 (Figure 3C). Over the 1-hour stimulation, both KP54 and MVT-602 displayed a sustained response over time as quantitated by the AUC and at each time frame. However, there was no difference in the sustained signal profiles between KP54 and MVT602 (Figure 3D and Supplemental Videos 1 and 2). Overall, this suggests that while MVT-602 may exhibit a much higher potency at the KISS1R, it does not differ in its ability to alter the acute nor persistent $\mathrm{Ca}^{2+}$ signal profile over time when compared with KP54.
MVT-602 increases firing rate in GnRH neurons for a more prolonged duration than KP54. Targeted extracellular recordings of GFP-identified GnRH neurons were used to evaluate the effects of MVT-602 and KP54 on action potential firing rate. Following either peptide, 7 of 8 neurons responded with an at least $30 \%$ increase in firing. Individual recordings of neurons exposed to KP54 and MVT602 are presented in Figure 4, A and B, respectively, and the (mean \pm SEM) firing frequency over time after each peptide is presented in Figure 4C. Although the onset of effect was similar after each peptide, typically commencing within 1-2 minutes of treatment, the duration of the effect was markedly increased to a median of 115 
Table 2. Pharmacokinetic and pharmacodynamic responses of KP54 and MVT-602

\begin{tabular}{|c|c|c|c|c|}
\hline Pharmacokinetic response & KP54, $9.6 \mathrm{nmol} / \mathrm{kg}(n=9)$ & MVT-602, $0.01 \mathrm{nmol} / \mathrm{kg}(n=3)$ & MVT-602, $0.03 \mathrm{nmol} / \mathrm{kg}(n=3)$ & $P$ value \\
\hline$t_{1 / 2}, \mathrm{~h}$ (range) & $\begin{array}{c}1.68 \\
(1.31,1.78)\end{array}$ & $\begin{array}{c}2.02 \\
(1.58,2.26)\end{array}$ & $\begin{array}{c}1.78 \\
(1.60,2.11)\end{array}$ & 0.22 \\
\hline$t_{\text {max' }}, \mathrm{h}$ (range) & $\begin{array}{c}1.00 \\
(0.91,1.22)\end{array}$ & $\begin{array}{c}0.42 \\
(0.32,0.46)\end{array}$ & $\begin{array}{c}0.36 \\
(0.34,0.37)\end{array}$ & $\begin{array}{l}0.0004 \\
(0.02)\end{array}$ \\
\hline$C_{\max }, \mathrm{pmol} / \mathrm{L}$ (range) & $\begin{array}{c}8855 \\
(7207,14,821)\end{array}$ & $\begin{array}{c}8.11 \\
(7.78,10.17)\end{array}$ & $\begin{array}{c}29.49 \\
(28.15,29.53)\end{array}$ & $\begin{array}{c}<0.0001 \\
(0.01)\end{array}$ \\
\hline
\end{tabular}

Pharmacodynamic response on LH $\quad$ LH rise episode 1 LH rise episode 2 LH rise episode 1 LH rise episode 2 LH rise episode 1 LH rise episode 2

\begin{tabular}{|c|c|c|c|c|c|c|c|}
\hline \multirow[b]{2}{*}{ Time of start of episode (h) } & \multicolumn{2}{|c|}{$(n=9)$} & \multicolumn{2}{|c|}{$(n=9)$} & \multicolumn{2}{|c|}{$(n=9)$} & \multirow[b]{2}{*}{$\begin{array}{l}0.0036 \\
(0.0036)\end{array}$} \\
\hline & $\begin{array}{c}2.44 \\
(1.96-3.32)\end{array}$ & NA & $\begin{array}{c}4.63 \\
(2.93-8.38)\end{array}$ & $\begin{array}{c}19.33 \\
(17.89-21.26)\end{array}$ & $\begin{array}{c}5.85 \\
(3.89-9.38)\end{array}$ & $\begin{array}{c}18.34 \\
(17.52-19.84)\end{array}$ & \\
\hline Duration of ascent of $L H$ (h) & $\begin{array}{c}2.07 \\
(1.63-3.63)\end{array}$ & NA & $\begin{array}{c}4.24 \\
(2.88-10.31)\end{array}$ & $\begin{array}{c}0.21 \\
(0.08-2.24)\end{array}$ & $\begin{array}{c}5.72 \\
(2.63-17.57)\end{array}$ & $\begin{array}{c}1.70 \\
(0.27-3.97)\end{array}$ & $\begin{array}{c}0.0085 \\
(0.01)\end{array}$ \\
\hline Duration of decay in LH (h) & $\begin{array}{c}2.36 \\
(2.04-4.14)\end{array}$ & NA & $\begin{array}{c}23.03^{* * *} \\
(10.14-33.29)\end{array}$ & $\begin{array}{c}4.24 \\
(2.88-10.31)\end{array}$ & $\begin{array}{c}15.98^{* *} \\
(6.14-18.21)\end{array}$ & $\begin{array}{c}11.66 \\
(4.96-16.2)\end{array}$ & $\begin{array}{c}0.0002 \\
\left({ }^{* * *} 0.0003,{ }^{* *} 0.005\right)\end{array}$ \\
\hline Time of maximal LH (h) & $\begin{array}{c}4.70 \\
(4.03-5.31)\end{array}$ & NA & $\begin{array}{c}19.69 \\
(11.72-34.81)\end{array}$ & $\begin{array}{c}21.86^{* *} \\
(18.83-27.37)\end{array}$ & $\begin{array}{c}15.14 \\
(10.47-20.73)\end{array}$ & $\begin{array}{c}21.42^{* * *} \\
(20.27-23.03)\end{array}$ & $\begin{array}{c}0.0002 \\
\left({ }^{* *} 0.0015,{ }^{* * *} 0.0005\right)\end{array}$ \\
\hline AUC of LH (IU·h/L) & & & & $\begin{array}{l}3^{*} \\
33.0)\end{array}$ & $\begin{array}{r}1 \\
(96\end{array}$ & & $\begin{array}{c}0.0058 \\
\left({ }^{*} 0.01,{ }^{* *} 0.029\right)\end{array}$ \\
\hline
\end{tabular}

Median (range) is presented for pharmacokinetic (PK) parameters and median (IQR) for pharmacodynamic (PD) parameters. To extract PK parameters for KP54 and MVT-602, we used a PK model incorporating drug absorption and elimination (51). To extract information regarding LH dynamics in response to administration of KP54 and MVT-602, we used a parametric model describing a superposition of 2 episodes of LH rise. As some LH rises had distinct phases of increase in serum LH after MVT-602, the model generated parameters for both a first-phase increase commencing within the first 10 hours of administration (LH rise episode 1), and a second-phase increase commencing after 10 hours (LH rise episode 2). Time of start indicates the time of onset of rise in LH. Amplitude of LH response refers to absolute LH levels. The area under the curve (AUC) of LH incorporates both LH rise episode 1 and episode 2. Time of start of increase in LH, duration of ascent, duration of decay, and time of maximal LH were compared using LH rise episode 1 , whereas amplitude of LH was compared using the greater of the $2 \mathrm{LH}$ amplitudes. Groups were compared by Kruskal-Wallis test with post hoc Dunn's test. If the initial $P$ value is less than 0.05 , the post hoc $P$ value is presented in parentheses and corresponds to groups in bold (and also by number of asterisks if to more than 1 group).

minutes after MVT-602 as compared with 55 minutes after KP54 $(P=0.0012)$ (Figure 4D). The firing frequency of all GnRH neurons had returned to baseline firing activity by 3 hours. This is unlikely to represent a loss of viability of these cells, as recordings lasting as long as 6 hours have been reported $(30,31)$. The peak firing frequency was higher after MVT-602 than after KP54 $(P=0.007)$ (Figure $4 \mathrm{E})$. These data are in keeping with our human clinical data, which show that MVT-602 induces a significantly prolonged duration of LH release compared with KP54.

MVT-602 in the healthy follicular phase, PCOS, and HA. Plasma MVT-602 levels were similar in both healthy and oligo/anovulatory women after they received a s.c. bolus of $0.03 \mathrm{nmol} / \mathrm{kg}$ of MVT$602(P=0.76)$ (Figure 5A). The rise in LH levels after MVT-602 was similar in women with PCOS to those in the healthy follicular phase (Figure 5B and Table 3). However, in women with HA, LH levels increased sooner after MVT-602 (time of first peak: 6.2 hours in HA vs. 15.1 hours in the healthy follicular phase; $P=0.008$ ) (Figure $5 \mathrm{~B}$ and Table 3). The total AUC of LH increase did not differ between these groups (Figure 5C). Furthermore, the increase in FSH levels following MVT-602 was particularly marked in women with $\mathrm{HA}(P=$ $0.0011)$ (Figure 5D) with a corresponding greater increase in estradiol $(P=0.023)$ (Figure 5E). Raw values for serum hormone levels after MVT-602 in the healthy follicular phase, PCOS, and HA are presented in Supplemental Figure 3.
Effect of estrogen supplementation on the response to MVT-602 in the healthy follicular phase. As the sex steroid milieu at the time of administration is known to influence the response to kisspeptin (8), we sought to determine the impact of estrogen supplementation on the response to MVT-602 in women. To do this, we examined the impact of increasing estradiol levels at the time of MVT-602 injection in the healthy follicular phase by applying a 200- $\mu \mathrm{g} / \mathrm{d}$ estradiol patch from 24 hours before administration of MVT-602 to achieve levels similar to those observed in the preovulatory phase of the menstrual cycle. The peak rise in LH after MVT-602 was increased from $7.5 \mathrm{IU} / \mathrm{L}$ to $24.4 \mathrm{IU} / \mathrm{L}$ following estradiol pretreatment $(P<$ 0.0001 ) (Figure 6, A and C). The peak rise in FSH was also greater, increasing from $1.7 \mathrm{IU} / \mathrm{L}$ to $7.6 \mathrm{IU} / \mathrm{L}(P<0.0001)$ (Figure 6, B and $D)$. However, the time of peak gonadotropin rise was not altered by estradiol supplementation. Individual and grouped hormonal profiles after MVT-602 with and without estradiol pretreatment are presented in Supplemental Figure 4.

\section{Discussion}

MVT-602 is a nanopeptide KISS1R agonist developed to have increased stability, potency, and water solubility (20). To our knowledge, this is the first study to determine its PD and PK profiles in healthy women, and to directly compare these properties with those of native KP54, and with those in women with the 2 
A

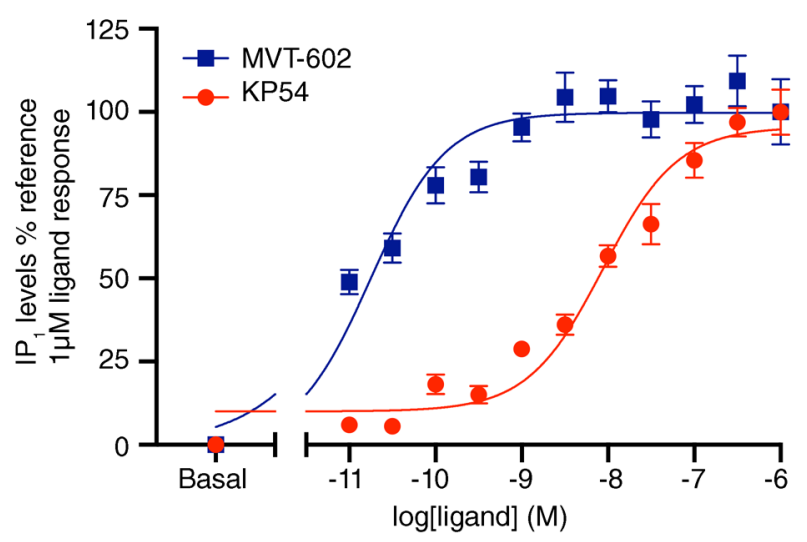

B

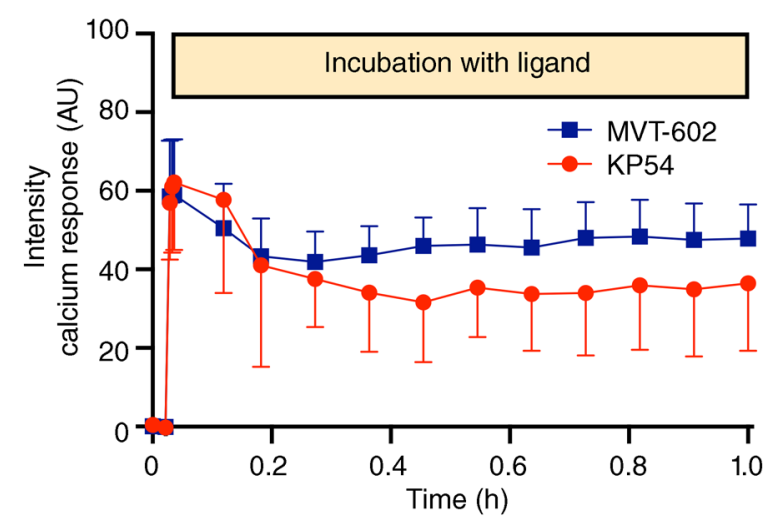

C

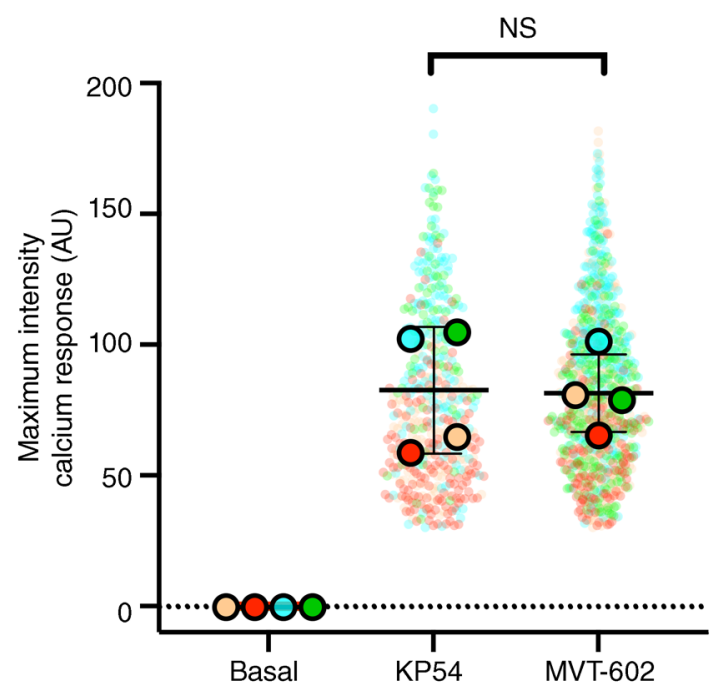

D

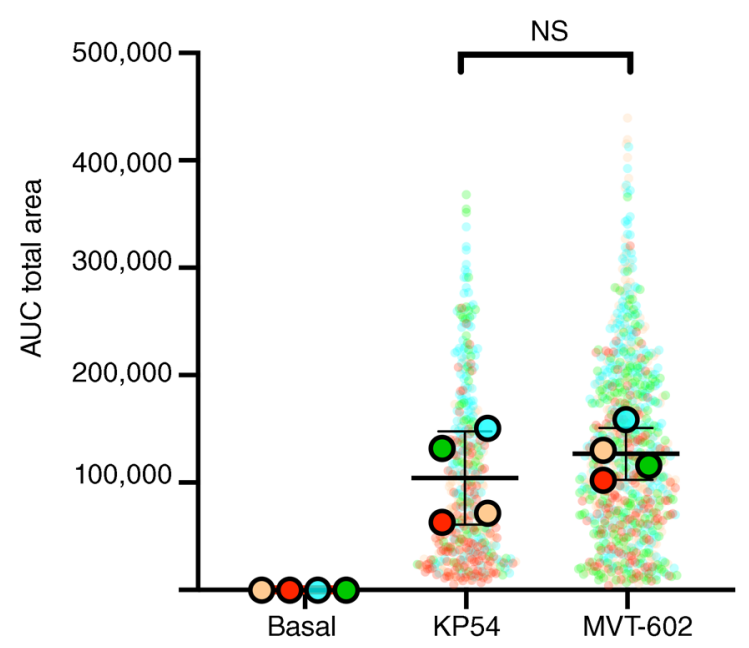

Figure 3. Effect of KP54 and MVT-602 on KISS1R-mediated $\mathbf{G}_{\mathbf{q}} / 11$ signaling via IP $\mathbf{P}_{1}$ and $\mathrm{Ca}^{2+}$ activation. (A) Intracellular levels of $\mathrm{IP}_{1}$ accumulation at varying concentrations of KP54 or MVT-602 following 45 minutes of stimulation in HEK293 cells expressing FLAG-KISS1R. Data represent mean ( \pm SEM) of $n=4$ independent experiments conducted in triplicate wells and are normalized as a percentage of ligand response. (B) Intracellular Ca ${ }^{2+}$ levels measured by Fluo-4AM $\mathrm{Ca}^{2+}$ indicator dye and live confocal microscopy. Intensity profile produced following $10 \mathrm{nM}$ stimulation with KP54 (red) or MVT-602 (blue) over 1 hour of chronic stimulation in cells transiently transfected with FLAG-KISS1R of $n=4$ independent experiments conducted in duplicate wells. Data are shown following subtraction of the average background intensity for each cell as described in Methods. (C and D) Maximum intensity (C) and total area under the curve (AUC) (D), calculated from data depicted in B following ligand treatments over 1 hour of chronic stimulation. Data show individual cell analysis (total number of cells: basal, $n=1324$; KP54, $n=472$; MVT-602, $n=852$ ) overlaid with the mean ( \pm SD) values of $n=4$ independent experiments conducted in duplicate wells. Cells attributed to each biological repeat are shown in the corresponding color. There were no significant differences between KP54 and MVT-602 following analysis by 2-tailed, unpaired Student's $t$ test.

most common causes of oligo/anovulatory infertility, PCOS and HA. Both MVT-602 and KP54 induced a similar peak LH amplitude, consistent with their analogous mechanism of action via stimulation of KISS1R on hypothalamic GnRH neurons $(4,32)$. However, although PK properties were similar following s.c. bolus administration, MVT-602 produced a markedly prolonged PD effect compared with KP54 (time of peak LH: 21-22 hours vs. 4.7 hours). Accordingly, the AUC of LH was increased more than 4-fold after MVT-602 in comparison with KP54 (33), identifying MVT- 602 as a prospective therapeutic for the treatment of female reproductive disorders using KISS1R-targeted drugs.

Due to kisspeptin's action to directly stimulate hypothalamic GnRH neurons, there has been tremendous interest in the development of KISS1R agonists for the treatment of reproductive disorders (11). KP54 can be used to mature oocytes safely for IVF treatment (12), without causing the dangerous complication of ovarian hyperstimulation syndrome (OHSS), even in women at increased a priori risk of OHSS (13). Moreover, extending the duration of the LH response with a second dose of KP54 further improves oocyte maturation without increasing the occurrence of OHSS (34). Hypothalamic kisspeptin signaling is known to be obligatory for the occurrence of preovulatory gonadotropin surge $(35,36)$. The natural mid-cycle gonadotropin surge in women is triphasic, comprising a rapidly ascending limb lasting 14 hours, followed by a plateau lasting for 14 hours, and then a descending limb lasting for 20 hours (33). The most commonly used current trigger of oocyte maturation during IVF treatment is human chorionic gonadotropin (hCG), which has an excessive duration of action lasting more than 1 week and consequently is the major cause of OHSS (37). hCG provides only LH-like activity with- 
A

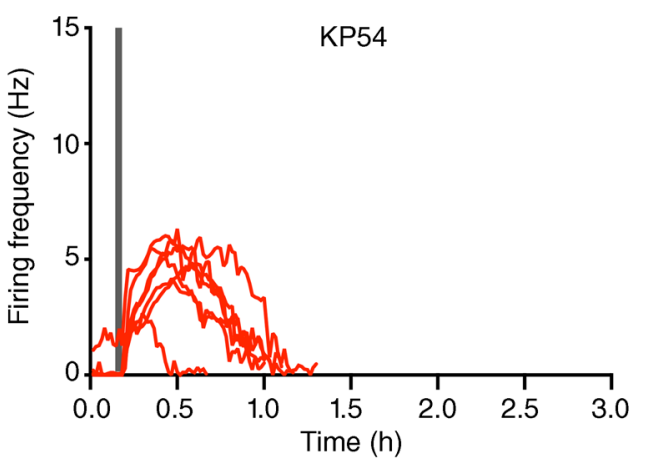

B

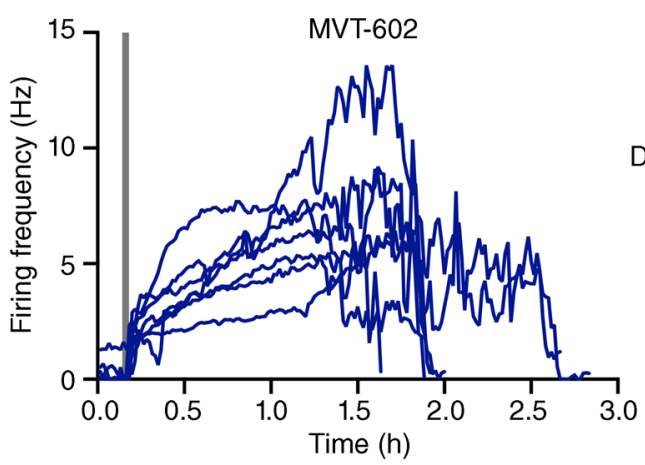

D

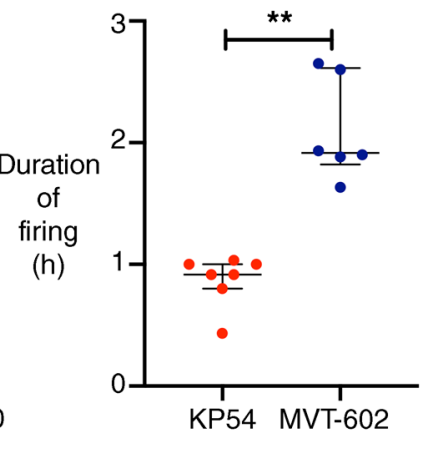

C

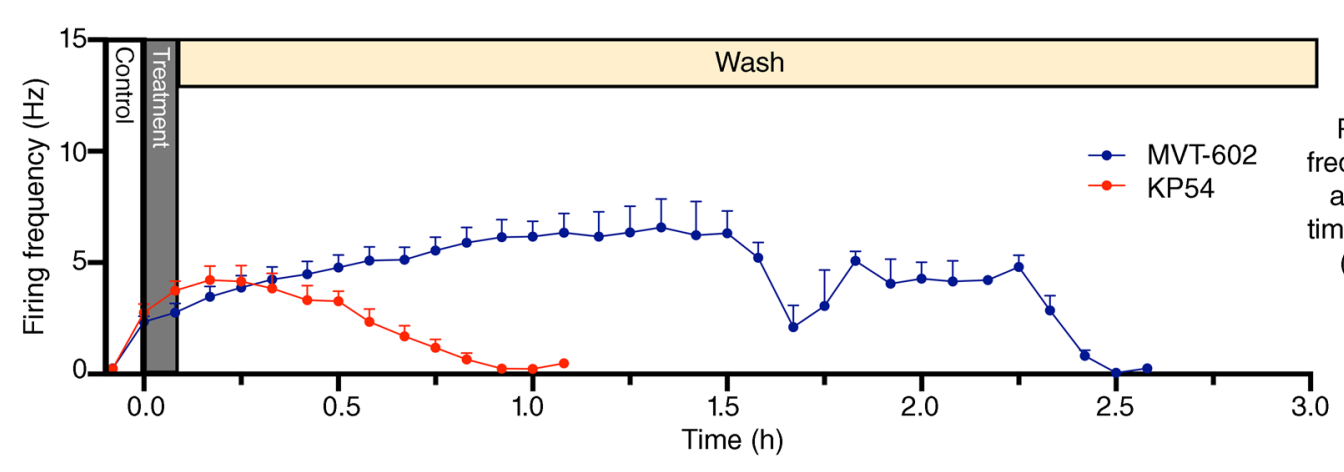

\section{E}

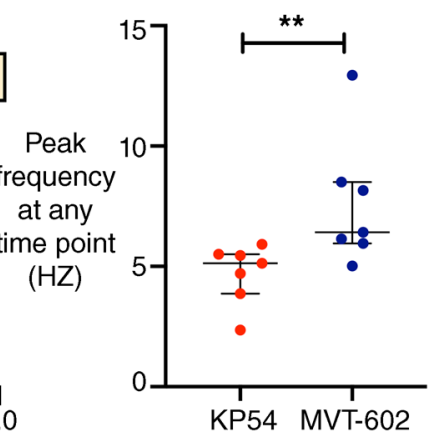

Figure 4. Targeted extracellular recordings of firing rate of GFP-identified GnRH neurons reveal that both KP54 and MVT-602 rapidly increase action potential firing rate, but the response to MVT-602 is more prolonged. Spontaneous basal activity (control) was recorded for 10 minutes, then either $10 \mathrm{nM}$ KP54 or 10 nM MVT-602 was bath-applied for 5 minutes, followed by a wash period of at least 40 minutes. (A and B) Firing frequency (Hz in 1-minute bins) of individual GnRH neurons versus time (hours) ( $n=7$ ) before, during (gray bar), and after exposure to either 10 nM KP54 (A) or 10 nM MVT-602 (B). (C) Mean ( \pm SEM) firing frequency of GnRH neurons ( $n=7$ per group, 5 -minute bins) over time (hours) before, during (gray bar), and after exposure to either 10 nM KP54 (red) or 10 nM MVT-602 (blue). Statistical analysis by mixed-effect model (restricted maximum likelihood) was truncated at 65 minutes because all KP54 cells had returned to baseline $(P=0.003)$. (D) Median (IQR) of duration of response (hours) of GnRH neurons maintaining a firing frequency greater than $1 \mathrm{~Hz}$ ( $n=7$ per group) after exposure to $10 \mathrm{nM} \mathrm{KP54}$ (red) or $10 \mathrm{nM}$ MVT-602 (blue). Groups were compared by the Mann-Whitney $U$ test (** $P=$ 0.0012 ). (E) Median (IQR) of the peak firing frequency $(\mathrm{Hz})$ of $\mathrm{GnRH}$ neurons ( $n=7$ per group) at any time point after exposure to $10 \mathrm{nM} \mathrm{KP54}(\mathrm{red})$ or $10 \mathrm{nM}$ MVT-602 (blue). Groups were compared by the Mann-Whitney $U$ test $\left({ }^{* *} P=0.007\right)$.

out the accompanying FSH rise that accompanies the natural mid-cycle LH surge $(33,38,39)$. Furthermore, activation of $\mathrm{LH}$ receptor by hCG results in different intracellular signaling than activation by LH, reflecting the physiological role of hCG in the maintenance of pregnancy rather than in the induction of oocyte maturation/ovulation (37). While GnRH agonists induce rises in both $\mathrm{LH}$ and FSH, the amplitude of LH reached is supraphysiological ( $150 \mathrm{IU} / \mathrm{L})$, and the duration of the LH surge is shorter (peak LH at 4-6 hours) $(12,33,38-40)$. Thus, the endocrine profile of MVT-602 is distinct and is likely to be more similar to that of the natural mid-cycle gonadotropin surge than that induced by currently available triggers of oocyte maturation. Further studies assessing whether MVT-602's more physiological endocrine profile translates to improved clinical outcomes during IVF treatment would be of great interest.

Kisspeptin also offers potential for the treatment of functional hypogonadal disorders associated with hypothalamic dysfunction such as diabetes-induced secondary hypogonadism (41) and age-related hypogonadism (42). While the treatment of functional hypogonadal disorders using kisspeptin could thus repre- sent an attractive therapeutic intervention, these would require chronic stimulation protocols. However, chronic administration of KP54 can result in tachyphylaxis (43), which is most commonly encountered in the context of frequent high-dose administration (44). A continuous s.c. infusion of MVT-602 in men induced tachyphylaxis at a minimum steady-state concentration of at least $228 \mathrm{pg} / \mathrm{mL}(186 \mathrm{pmol} / \mathrm{L})(23)$. It is notable that the peak circulating MVT-602 level in the present study in women was about 6-fold lower ( $<30 \mathrm{pmol} / \mathrm{L})$ after a s.c. bolus of MVT-602 (0.03 $\mathrm{nmol} / \mathrm{kg}$ ) (Table 2). Furthermore, sensitivity to kisspeptin can modulate the susceptibility to tachyphylaxis; i.e., the same dose of KP54 administered twice daily induced tachyphylaxis in women with HA (15), but not when administered in the healthy follicular phase (45). As women in the follicular phase are less sensitive to MVT-602, one could speculate that higher circulating levels may be needed to induce tachyphylaxis in comparison with men, whereas even lower doses could be chosen to achieve continued gonadotropin stimulation while avoiding tachyphylaxis in most women with HA. Indeed, while MVT-602 can also induce tachyphylaxis if given continuously as a high-dose s.c. infusion (23), its 
A

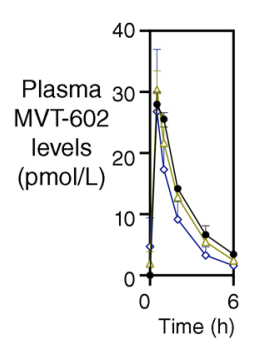

B

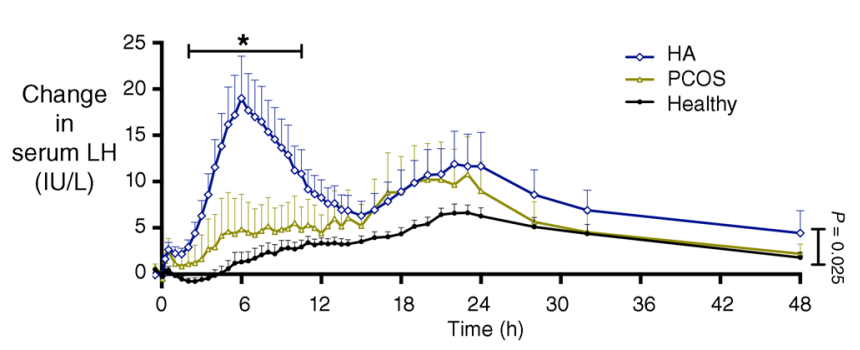

C

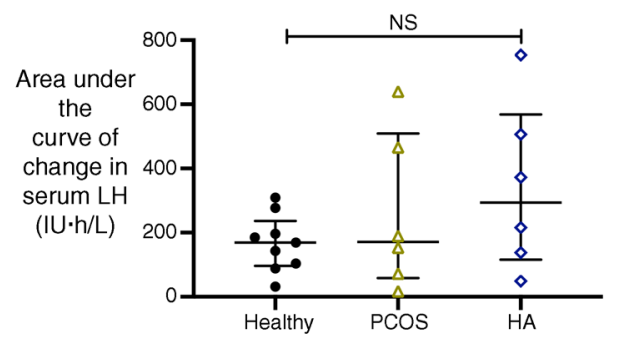

D

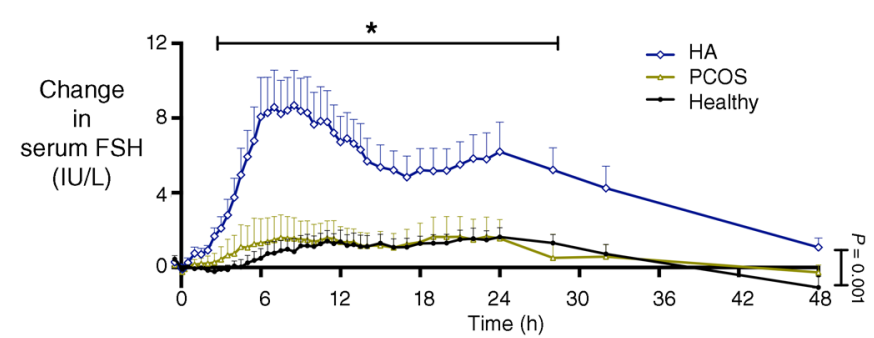

E

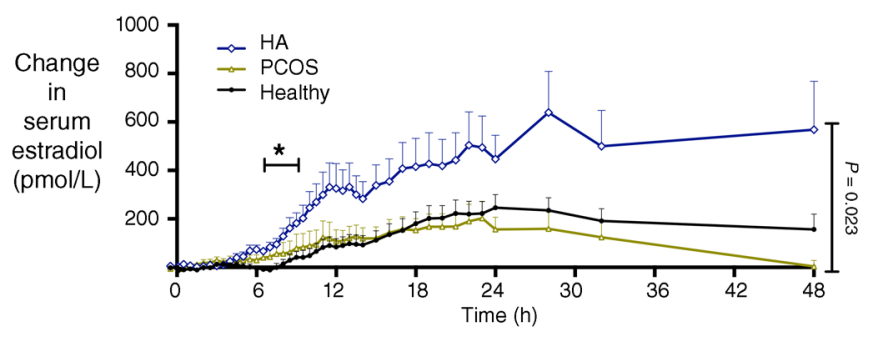

Figure 5. Clinical studies of MVT-602 in women with oligo/anovulatory disorders. (A) Mean ( \pm SEM) plasma concentration of MVT-602 (pmol/L) versus time (hours) in women receiving an s.c. bolus of $0.03 \mathrm{nmol} / \mathrm{kg}$ of MVT-602. Healthy women in the follicular phase are presented in black $(n=9)$, women with PCOS in green $(n=6)$, and women with HA in blue $(n=6)$. No significant difference was detected by 2-way ANOVA. (B, D, and E) Mean ( \pm SEM) change from baseline levels in serum LH (IU/L) (B), serum FSH (IU/L) (D), and serum estradiol (pmol/L) (E) in healthy women (black; $n=9$ ), women with PCOS (green; $n$ $=6$ ), and women with $\mathrm{HA}$ (blue; $n=6$ ) after receiving a single s.c. bolus of $0.03 \mathrm{nmol} / \mathrm{kg} \mathrm{MVT-602.} \mathrm{Groups} \mathrm{were} \mathrm{compared} \mathrm{by} 2$-way ANOVA. ${ }^{*} P=0.025$ for $\mathrm{LH},{ }^{*} P=0.001$ for FSH; ${ }^{*} P=0.02$ for estradiol. (C) Median (IQR) of modeled values of AUC of serum LH (IU.h/L) with $0.03 \mathrm{nmol} / \mathrm{kg} \mathrm{MVT-602} \mathrm{in} \mathrm{women} \mathrm{in} \mathrm{the}$ healthy follicular phase, women with PCOS, and women with HA. No significant difference was detected by Kruskal-Wallis test.

ability to induce a prolonged duration of gonadotropin elevation lasting about 48 hours after a single bolus injection could facilitate chronic stimulation protocols that use infrequent (e.g., every 2-3 days) low-dose bolus administration that could mitigate against tachyphylaxis (16). Conversely, the prolonged duration of action of MVT-602 on GnRH neuronal firing in vitro and on LH release in women, coupled with its increased potency, could indicate that tachyphylaxis may yet challenge continued stimulation with the use of MVT- 602 in a chronic protocol. Consequently, further studies investigating chronic administration protocols of MVT-602 are indicated to assess whether persistent stimulation can be achieved with low-dose intermittent administration.

Continuous exposure of the KISS1R to KP10 elicits a biphasic increase in intracellular $\mathrm{Ca}^{2+}$ signaling, with an acute first-phase response lasting 5 minutes, followed by a more sustained second-phase response lasting more than 30 minutes (46). KISS1R is a $G \alpha_{\mathrm{q}} / 11$-coupled receptor (27); however, it is also recognized that mechanisms in addition to $\mathrm{G}_{\alpha} / 11$ signaling can induce $\mathrm{LH} / \mathrm{GnRH}$ secretion, such as via the adaptor protein arrestin to activate ERK signaling $(47,48)$. In addition to its key role as an adaptor protein, arrestin also mediates GPCR internalization, and, notably, the persistent second wave in $\mathrm{Ca}^{2+}$ signaling after $\mathrm{KP} 10$ is dependent on KISS1R internalization (46). Here we report, for the first time to our knowledge, that continuous exposure to KP54, and MVT-602, also produced prolonged $\mathrm{Ca}^{2+}$ signaling responses, akin to KP10, which may suggest that receptor trafficking may also be involved in regulating signal kinetics from KP54 and MVT-602.
We compared MVT-602 with a dose of KP54 $(9.6 \mathrm{nmol} / \mathrm{kg})$ that is likely to induce a near-maximal response on LH during clinical studies $(9,12,13)$. The 500-fold increased potency of MVT-602 at the human KISS1R to induce IP signaling is consistent with the clinical data, in which a 300-fold lower dose of MVT- 602 produced similar elevations in gonadotropins compared with KP54. PK parameters for KP54 and MVT-602 were surprisingly similar. The half-life of KP54 reported in the present study (1.7 hours) is longer than that previously reported ( 0.5 hours) (8), which is likely attributable to the mode of administration in the present study (s.c. rather than i.v.). MVT- 602 has been reported to have a similar half-life (1.5-3.5 hours) in men using the same s.c. route of administration (23).

C6 is a Kiss1r agonist synthesized through modification of KP10, by addition of a serum albumin-binding motif and $\omega$-methylation of arginine to resist trypsin-like proteases (49). Thus, C6 was confirmed to have increased potency compared with KP10 in a calcium mobilization assay $\left(\mathrm{EC}_{50} 0.33\right.$ vs. $\left.2.6 \mathrm{nM}\right)(49)$. C6 has been studied in preclinical models but not in humans; intramuscular injection of $15 \mathrm{nmol}$ of C6 induced an LH rise that lasted about 12 hours in ewes in both breeding and nonbreeding seasons (49). C6 also increased gonadotropin release and triggered ovulation in alpine goats, suggesting that $\mathrm{C} 6$ could offer an alternative to pregnant mare serum gonadotropin in animal husbandry (50). Application of $\mathrm{C} 6$ at doses from 0.01 to $40 \mathrm{nM}$ stimulated action potential firing in approximately half of murine GnRH neurons tested (49). Once-daily i.p. administration of $\mathrm{C} 6$ for 5 days advanced puberty in mice (49). One could hypothesize that the reduced potency of C6 
Table 3. Pharmacokinetic profile and pharmacodynamic responses of serum LH to MVT-602 in healthy women, women with PCOS, and women with HA

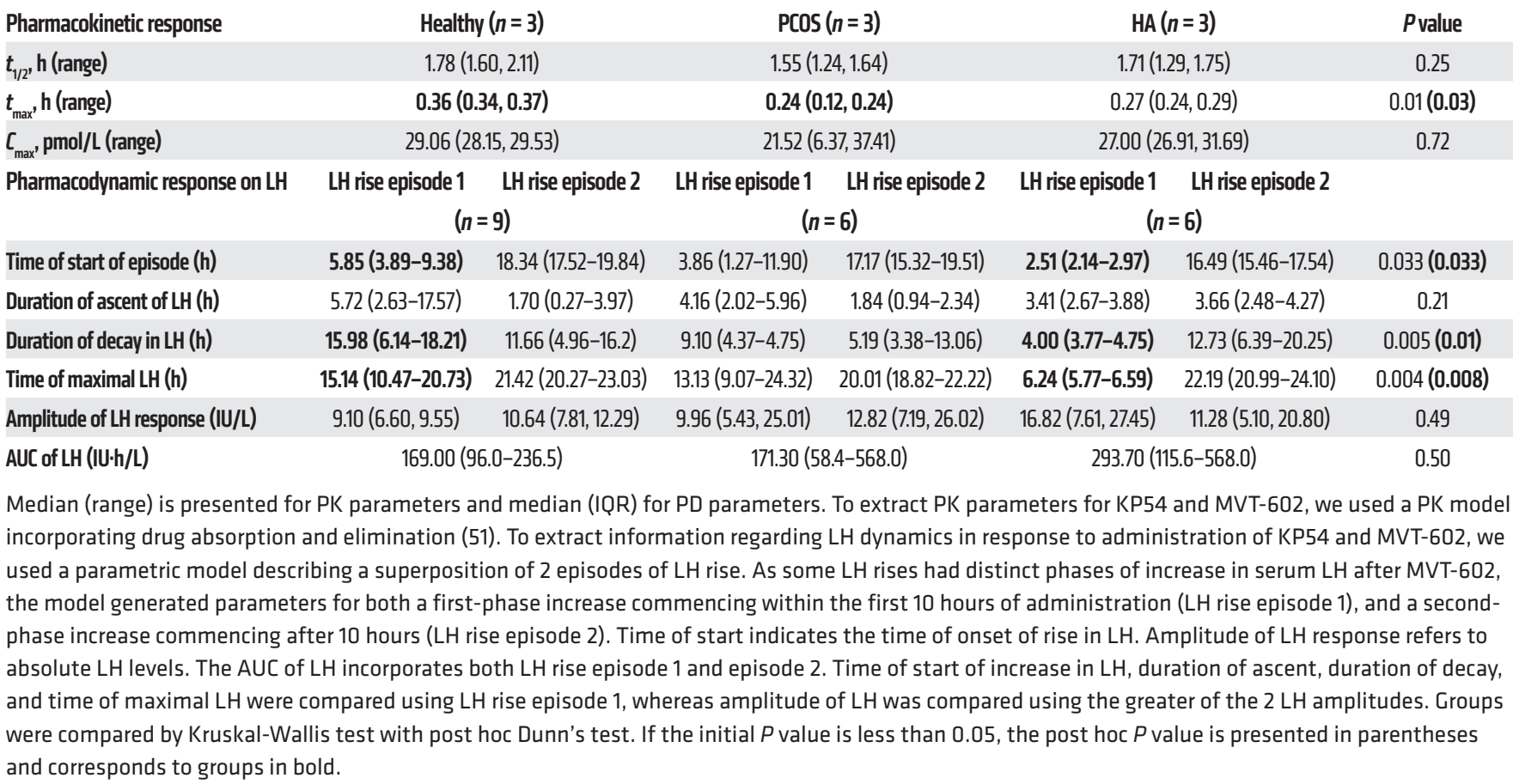

could theoretically reduce the chance of tachyphylaxis; however, the rise in LH was reduced after 5 days of daily administration, suggesting that C6 may also be susceptible to tachyphylaxis despite its reduced potency (49). C6 has yet to be tested in humans, and thus further studies would be needed to compare the endocrine profile of C6 with that of KP54 or MVT-602 in women.

Notably, there may be ligand-dependent differences in receptor regulation that cannot be recapitulated in a HEK293 cell model; thus it was also important to compare the effects of MVT-602 versus KP54 on GnRH neurons. Kisspeptin is a strong activator of action potential firing in GnRH neurons (30) and GnRH release $(51,52)$, with the latter dependent on both intra- and extracellular $\mathrm{Ca}^{2+}$ sources (52). Exposure of GnRH neurons in brain slices to only a short exposure of MVT-602 (5 minutes) elevated firing for a longer duration (115 vs. 55 minutes) and to a greater peak firing rate than an equimolar amount of KP54. These findings are consistent with the prolonged elevation of gonadotropin levels following MVT-602 compared with KP54 that we observed in women. There could be multiple mechanisms underlying the distinct kinetic profiles in firing rate between KP54 and MVT-602 following a brief exposure to GnRH neurons. Each ligand could have differential binding kinetics; ability to activate distinct signaling pathways, including signal crosstalk with other neuronal GPCRs; or altered ability for Kiss1r to be desensitized. In addition, it would be interesting to determine how these distinct firing rates, and signaling mechanisms, impact GnRH secretion and pulsatility.

It is interesting to consider the distinct durations of ligand exposure required for Kiss1r action during electrophysiological studies in GnRH neurons versus intracellular $\mathrm{Ca}^{2+}$ signaling stud- ies in HEK293 cells. Brief exposure to a Kiss1r ligand can induce persistent action potential firing in GnRH neurons, whereas sustained exposure of Kiss1r to the ligand is required to induce $\mathrm{Ca}^{2+}$ signaling in HEK293 cells. No previous study has investigated the effects of KP54 or MVT-602 either on the action potential firing in GnRH neurons or on intracellular $\mathrm{Ca}^{2+}$ signaling in HEK293 cells. Thus, there is no direct comparison with our data available in the literature. In response to KP10 (10-100 nM), however, phospholipase C (PLC) appears to be the major pathway for intracellular signaling in GnRH neurons (53). Exposure of GnRH neurons to $\mathrm{KP10}(10-100 \mathrm{nM})$ for 1-3 minutes elicits depolarization that lasts for at least 20 minutes (4); however, the number of GnRH neurons that responded to KP10 was reduced from $80 \%$ to $15 \%$ with pharmacological PLC antagonism and to $7 \%$ with $\mathrm{IP}_{3}$ antagonism (53). Intracellular $\mathrm{Ca}^{2+}$ studies in $\mathrm{GnRH}$ neurons find that exposure to $\mathrm{KP} 10$ (100 $\mathrm{nM})$ for about 3 minutes evoked an immediate rise in intracellular $\mathrm{Ca}^{2+}$ that lasted for 2-3 minutes before a sudden drop and a gradual return to basal concentrations (53). These data are congruous with our own findings in suggesting that the mechanisms contributing to the prolonged increase in firing rate of GnRH neurons may be independent of both persistent activation of Kiss1r and continued elevation of intracellular $\mathrm{Ca}^{2+}$. In summary, while experimental models evaluating both $\mathrm{GnRH}$ neuronal firing and intracellular $\mathrm{Ca}^{2+} / \mathrm{IP}_{1}$ signaling provide valuable insight into the differential effects of MVT-602 and KP54 at Kiss1r, there may be additional differences in downstream signaling molecules that may not have been captured. For example, it is possible that phosphorylation of a downstream molecule after Kiss1r activation with a much longer duration of action could enable GnRH neurons 
A

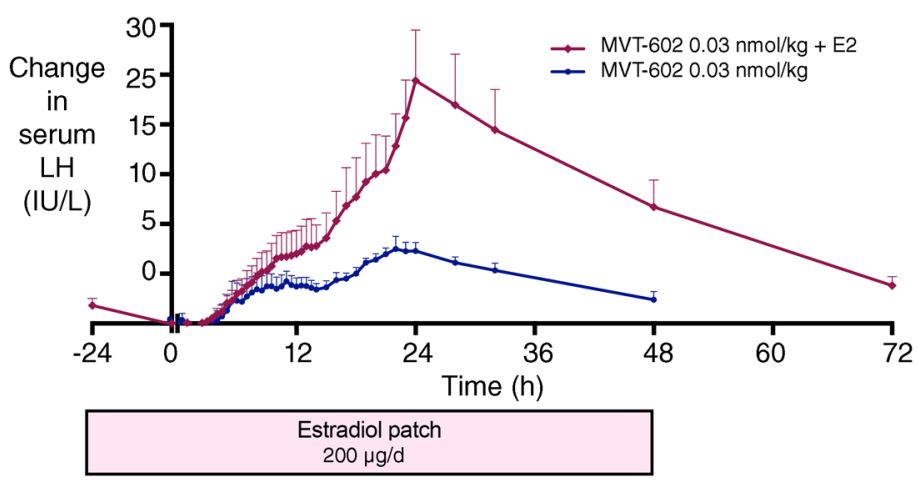

B

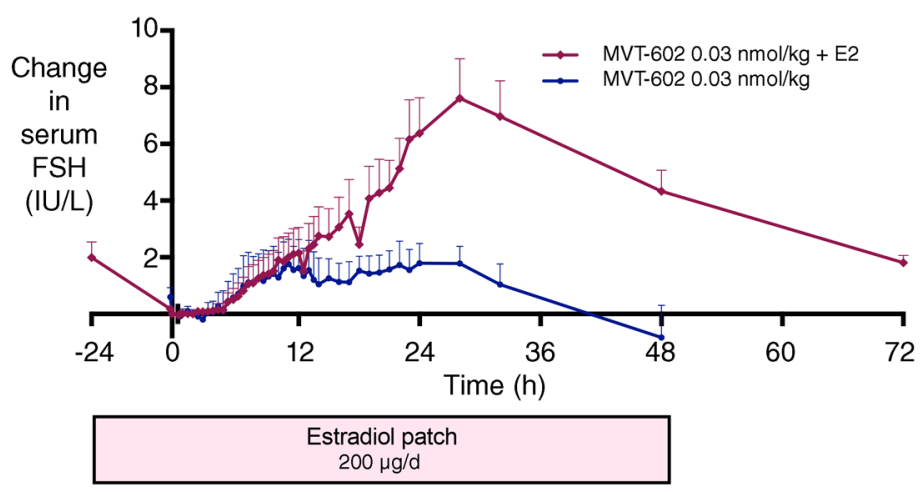

C

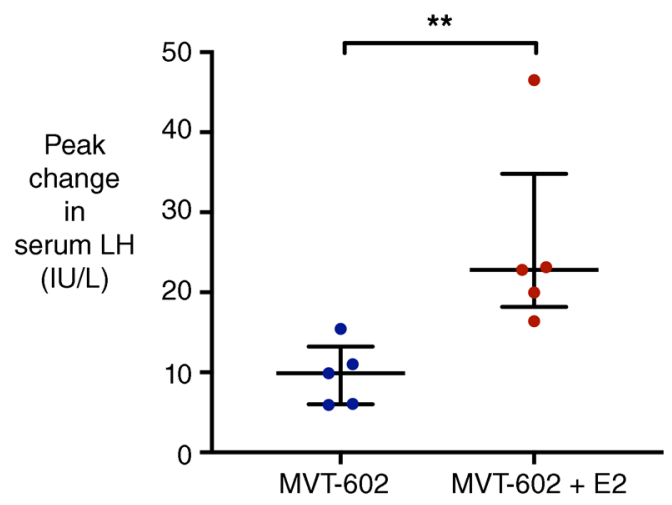

D

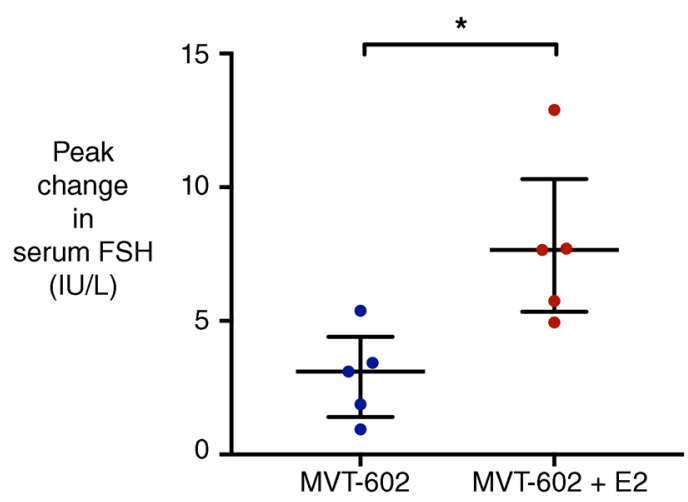

Figure 6. Effect of estradiol supplementation on response to MVT-602 in women during the healthy follicular phase. (A and B) Mean ( \pm SEM) change from baseline in serum LH (IU/L) (A) and serum FSH (IU/L) (B) in healthy women receiving a single s.c. bolus of $0.03 \mathrm{nmol} / \mathrm{kg} \mathrm{MVT-602} \mathrm{(blue),} \mathrm{and} \mathrm{in}$ women with estradiol supplementation ( $n=5$ per group) via a $200-\mu \mathrm{g} / \mathrm{d}$ transdermal patch applied from 24 hours before MVT-602 administration at time 0 hours and continued until time 48 hours (red). (C and D) Scatterplot of median (IQR) of maximal rise in serum LH (IU/L) (C) and serum FSH (IU/L) (D) in healthy women receiving a single s.c. bolus of $0.03 \mathrm{nmol} / \mathrm{kg}$ MVT-602 (blue), and in women with estradiol supplementation ( $n=5$ per group) via a $200-\mu \mathrm{g} / \mathrm{d}$ transdermal patch applied from 24 hours before MVT-602 administration at time 0 hours and continued until time 48 hours (red). Groups were compared by Mann-Whitney $U$ test $\left({ }^{*} P=0.016,{ }^{* *} P=0.0079\right)$.

to continue to fire without requiring the ongoing presence of the ligand at the receptor. By comparison, $\mathrm{IP} / \mathrm{Ca}^{2+}$ intracellular signaling experiments may provide a more direct portrayal of ongoing Kiss1r activation.

Interestingly, the rise in LH following MVT-602 during clinical studies was delayed for about 6 hours when studied in the healthy follicular phase. Similarly, KP54 also has a similar, albeit briefer, plateau in LH rise between 0.5 and $1.5 \mathrm{~h}$ before a further LH rise to its peak at 4.9 hours, suggesting that the observed delay in LH rise could be a KISS1R-mediated effect. However, the onset of the firing response following both ligands was similarly rapid during electrophysiological GnRH neuron studies, indicating that factors preceding receptor occupancy, such as the time needed for gaining access across the bloodbrain barrier (BBB), are more likely to underlie this delay than deferred activation after binding to KISS1R. Indeed, MVT-602 was selected for its hydrophilic properties (20), which could conceivably alter its ability to cross the BBB, whereas KP54 is considered to cross the $\mathrm{BBB}(54,55)$. Interestingly, the delay in LH rise was not apparent in women with HA, nor in men (23), suggesting that such a factor would have to plausibly differ between these physiological states.

Another possible contributor to the differential onset of $\mathrm{LH}$ increase is differences in the sex hormone milieu at the time of administration. The LH rise following KP54 is recognized to be modified by the sex hormone milieu at the time of administration, being markedly increased in the preovulatory phase of the menstrual cycle when estradiol levels are highest (9). Correspondingly, estradiol enhances $\mathrm{GnRH}$ neuron firing response to kisspeptin in a model of the preovulatory phase (30) and increases expression of Kiss1r in GT1-7 GnRH neurons (56). Thus, we examined the impact of artificially increasing ambient estradiol levels in the healthy follicular phase from 24 hours before the time of administration of MVT-602 using estradiol patches to achieve estradiol levels analogous to those encountered during the preovulatory phase. We observed that peak gonadotropin ris- 
es were markedly increased more than 3 -fold after estradiol pretreatment; however, the time course of LH increase did not differ from that in the control healthy follicular phase. This observation was consistent with the more than 4 -fold increased $\mathrm{LH}$ response to an s.c. bolus of KP54 (6.4-12.8 nmol $/ \mathrm{kg})$ after controlled ovarian stimulation, in which estradiol levels are markedly elevated, compared with the healthy follicular phase $(9,12,13)$. Conceivably, administration of the estradiol patch 24 hours before MVT602 administration could allow sufficient time for a switch from negative to positive feedback at $\mathrm{GnRH}$ neurons and/or the pituitary and thus augment the $\mathrm{LH}$ response (57).

We also determined the endocrine profile induced by MVT602 in the 2 most common oligo/anovulatory disorders causing subfertility, namely PCOS and HA (24-26). The MVT-602induced increase in LH level occurred sooner in women with HA in comparison with the healthy follicular phase. Levels of MVT-602 itself did not differ, suggesting that the advanced LH response could not be attributed to a difference in PK parameters, at least in the circulation. Notably, in order to ensure that women with PCOS had a more uniform endocrine milieu, most women with PCOS received a 7-day course of progesterone to induce a withdrawal bleed before each study visit, whereas healthy women and women with HA did not. Consequently, it is possible that exposure to administered progesterone in the previous cycle could have negatively affected reproductive hormone levels in women with PCOS (58). Disturbance of LH pulsatility in HA is associated with reduced energy availability (<30 kcal per kg of lean body mass) (59). Kisspeptin expression is reduced and Kiss1r expression is increased in the hypothalamus of a rodent model deprived of nutrition for 72 hours (3). Thus, our findings could putatively be explained by a greater abundance of KISS1R in HA leading to an exaggerated gonadotropin rise. Indeed, the LH rise after exogenous KP54 is increased in women with HA in comparison with healthy women in the follicular phase (15). MVT-602 induced an exaggerated and early rise in $\mathrm{LH}$ in women with $\mathrm{HA}$, and some women also appeared to have a distinct secondary rise in LH. Notably, the rise in FSH was particularly pronounced in women with $\mathrm{HA}$, and it is possible that the resultant enhanced estradiol level following MVT-602 administration to women with HA (Figure 5E) could exceed the threshold to induce a switch from negative to positive estradiol feedback and thus prompt a secondary LH rise in these women. Moreover, the marked FSH response coupled with the prolonged duration of action raises the prospect of using MVT-602 as a sole ovulation induction agent in women with HA.

In summary, we delineate the endocrine profile of the KISS1R agonist MVT-602 in healthy women and those with PCOS and HA for the first time to our knowledge. MVT-602 induced more prolonged stimulation of the reproductive axis via hypothalamic GnRH neurons than is possible with native KP54. Our in vitro data suggest that this prolonged action of MVT-602 compared with KP54 is due to its increased potency on intracellular $\mathrm{Ca}^{2+}$ release and extended duration of stimulation of GnRH neurons. Taken together, our clinical and mechanistic data identify MVT-602 as a prospective therapeutic agent for the treatment of female reproductive disorders.

\section{Methods}

\section{Peptides}

Kisspeptin-54 (KP54) was synthesized and purified by Bachem (Bachem Holding AG). Sterile vials of KP54 were produced by Bachem (Clinalfa, Bachem Distribution Services $\mathrm{GmbH}$ ). Both products were prepared according to good manufacturing practice (GMP). Vials of freeze-dried KP54 were stored at $-20^{\circ} \mathrm{C}$ and reconstituted in $0.9 \%$ saline as described previously $(8,9)$.

MVT-602 (GMP grade) was provided by Myovant Sciences Ltd. as a clear sterile solution $(3.5 \mathrm{~mL})$ in vials containing $0.2 \mathrm{mg}(163.2$ $\mathrm{nmol}$ ) per $2 \mathrm{~mL}$ (100 ppm) for use in the study. For lower doses, vials were diluted using $5 \%$ dextrose for injection.

\section{Clinical studies}

Study protocol. Ethical approval for this study was granted by the West London Research Ethics Committee, London, UK (reference 12/LO/0507), and participants provided written informed consent. The study was conducted in accordance with the Declaration of Helsinki. Twelve healthy women, 6 women with HA, and 6 women with PCOS were recruited via newspaper advertisements.

Healthy ovulatory women aged $18-35$ years, with a menstrual cycle length less than 35 days, BMI $18-30 \mathrm{~kg} / \mathrm{m}^{2}$, not taking any medications or hormonal contraception, were invited to take part in the study. PCOS was defined according to the 2018 international PCOS guidelines (24), and women were diagnosed with HA in accordance with Endocrine Society guidelines (25). All women underwent detailed medical history, medication history, menstrual history, and clinical examination. The following blood tests were assessed during the screening visit: full blood count, renal function, liver function, bone profile, thyroid hormone profile, luteinizing hormone (LH), follicle-stimulating hormone (FSH), estradiol, progesterone, androstenedione, dehydroepiandrosterone, testosterone, sex hormone-binding globulin (SHBG), prolactin, and anti-Müllerian hormone.

All study visits were carried out at the Clinical Research Unit, Imperial College Healthcare NHS Trust. The study was single-blinded, and the order of receiving the intervention was decided using an online algorithm (www.random.org). All study visits were commenced in the morning $(\sim 8 \mathrm{am})$, and participants could eat and drink nonalcoholic beverages ad libitum before and during each study visit. Pregnancy was excluded using a urine pregnancy test at the beginning of each study visit. Blood pressure and pulse rate were recorded throughout each study visit and occurrence of any symptoms recorded for the week following each intervention. Details of the preliminary dose-finding study for MVT-602 are presented in Supplemental Methods.

Comparison of MVT-602 with KP54 in the healthy women. Following analysis of the preliminary dose-finding study, 2 doses of MVT-602, 0.03 and $0.01 \mathrm{nmol} / \mathrm{kg}$, were selected for comparison with KP54 $(9.6 \mathrm{nmol} / \mathrm{kg})$ or $0.9 \%$ saline vehicle in the early follicular phase of a further 9 healthy women. This dose of KP54 $(9.6 \mathrm{nmol} / \mathrm{kg})$ was selected because it induces a near-maximal gonadotropin rise for KP54 and safely induces oocyte maturation during IVF treatment $(9,12,13)$. Each woman received the following 4 interventions in random order: $0.01 \mathrm{nmol} / \mathrm{kg}$ MVT-602, 0.03 $\mathrm{nmol} / \mathrm{kg}$ MVT-602, $9.6 \mathrm{nmol} / \mathrm{kg} \mathrm{KP54}$, and 0.9\% saline, during 
the early follicular phase (days 1-4) in 4 different menstrual cycles (with only 1 intervention administered per menstrual cycle). Following KP54 and 0.9\% saline, serum reproductive hormone levels ( $\mathrm{LH}, \mathrm{FSH}$, estradiol, and progesterone) were measured every 5-15 minutes for the first 30 minutes, and then every 30 minutes until 10 hours, and additionally at 24 hours. Following MVT-602, the duration of blood sampling every 30 minutes for measurement of reproductive hormones was extended to 24 hours, with additional samples at 28, 32, and 48 hours (see Figure 1 for study protocol).

Comparison of the gonadotropin response to MVT-602 in healthy women in the follicular phase, women with PCOS, and women with HA. Six women with HA and 6 women with PCOS received a single s.c. dose of either $0.03 \mathrm{nmol} / \mathrm{kg} \mathrm{MVT-602,} 9.6 \mathrm{nmol} / \mathrm{kg} \mathrm{KP54}$, or $0.9 \%$ saline vehicle in each study visit. All women in this study followed the same protocol as described above. Anovulatory women with PCOS had menses induced with an oral course of progesterone (10 mg of medroxyprogesterone twice daily for 1 week) and had their study visits on days 1-4 after their withdrawal bleed. One woman with PCOS had spontaneous menstrual bleeding every 6 weeks and thus did not require progesterone induction. Women with HA did not have progesterone induction, as this was unlikely to result in a withdrawal bleed, but had study visits scheduled to occur once per month to ensure consistent treatment washout times.

The effect of estrogen supplementation on the response to the KISS1R agonist MVT-602. To investigate the effect of sex steroid milieu on the response to MVT-602, 5 healthy women in the early follicular phase had a transdermal estradiol patch $(200 \mu \mathrm{g} / \mathrm{d})$ applied 24 hours before the start of each study visit and continued for 72 hours (60). A single s.c. bolus of $0.03 \mathrm{nmol} / \mathrm{kg}$ MVT-602 was administered 24 hours after the initiation of estradiol treatment. Serum reproductive hormone levels ( $\mathrm{LH}, \mathrm{FSH}$, estradiol, and progesterone) were measured every 5-15 minutes for the first 30 minutes, and then every 30 minutes until 24 hours, with additional samples at 28, 32, 48, and 72 hours after MVT-602. Details of hormone assays are presented in Supplemental Methods.

Statistics. Statistical analyses were conducted using GraphPad Prism 8 (GraphPad Software). Parametrically distributed continuous variables were reported as mean $\pm \mathrm{SD}$, whereas skewed continuous variables were summarized using median (IQR). Parametrically distributed variables were compared using unpaired 2-tailed Student's $t$ test (2 groups) or 1-way ANOVA (multiple groups) with post hoc Tukey's test. Nonparametrically distributed variables were compared using Mann-Whitney $U$ test (2 groups) or Kruskal-Wallis test with post hoc Dunn's test (multiple groups) as appropriate. Changes in gonadotropin levels over time were compared by 2-way repeated-measures ANOVA with post hoc Tukey's test.

PK model to characterize PK parameters. To extract pharmacokinetic (PK) parameters for KP54 and MVT-602, we used a PK model incorporating drug absorption and elimination (61). This allowed capture of any delayed absorption as reflected in the measured plasma concentrations of MVT-602 and KP54 after s.c. administration of the peptides. The model describes drug transfer from the site of administration to central circulation as a first-order process with rate constant $1 / \tau_{\mathrm{a}}$. Clearance of the drug from central circulation is also modeled as a first-order process with rate $1 / \tau_{c}$. The equation describing peptide plasma concentration, $C(t)$, as a function of time is:

$$
C(t)=\frac{C_{\text {max }}\left(e^{-t / \tau_{a}}-e^{-t / \tau_{c}}\right)}{\left(e^{-T_{\text {peak }} / \tau_{a}}-e^{-T_{\text {peak }} / \tau_{c}}\right)}
$$

(Equation 1)

where $C_{\max }$ is the maximum peptide plasma concentration, and:

$$
\mathrm{T}_{\text {peak }}=\frac{\tau_{a} \tau_{c}}{\tau_{a}-\tau_{c}} \log \left(\tau_{a} / \tau_{c}\right)
$$

(Equation 2)

is the time the maximum is attained. To infer parameter estimates, we assumed that each model parameter $\theta\left(C_{\max }, \tau_{\mathrm{c}}, \tau_{\mathrm{a}}\right)$ has a Gaussian distribution within different groups (healthy, PCOS, HA), i.e., $N\left(\mu_{\theta, \text { group }}, \sigma_{\theta, \text { group }}\right)$. Additive Gaussian error was used with standard deviation informed by the coefficient of variation of the assays. As the pair of parameters $\tau_{\mathrm{c}}$ and $\tau_{\mathrm{a}}$ cannot be uniquely identified from our data (i.e., exchanging the values of the 2 parameters has no effect on the predicted plasma concentration), we report a quantity that is invariant to exchanges of these 2 parameters, namely $\log (2)$ $\times\left(\tau_{\mathrm{a}}+\tau_{\mathrm{c}}\right)$, corresponding to the half-life of the peptide in the system.

$P D$ model to characterize LH secretion. To extract information regarding LH dynamics in response to administration of KP54 and MVT-602, we used a parametric model describing a superposition of 2 episodes of LH rise.

Details of the mathematical form of the model used are presented in Supplemental Methods.

\section{Effect of MVT-602 and KP54 on human KISS1R signaling}

Details of reagents and DNA constructs and cell maintenance and transfection are presented in Supplemental Methods.

IP $P_{1}$ accumulation. HEK293 cells (ATCC CRL-1573) were plated into clear 96-well plates (Costar, Thermo Fisher Scientific) 24 hours after transfection. Then, 48 hours after plating, cells were stimulated at the indicated concentrations and time points in the presence of $50 \mathrm{mM} \mathrm{LiCl}$ in DMEM. The levels of $\mathrm{IP}_{1}$ were then determined on cell lysates using an IP-One Gq HTRF kit (Cishio, PerkinElmer) according to manufacturer instructions. Protein concentration of lysates was then determined by Bradford assay, and $\mathrm{IP}_{1}$ levels were normalized to protein concentration.

$\mathrm{Ca}^{2+}$ mobilization. HEK293 cells were plated into 35-mm imaging dishes (no. 1.5 coverslip; MatTek) 24 hours after transfection. Cells were incubated with Fluo-4AM for 30 minutes at $37^{\circ} \mathrm{C}, 5 \%$ $\mathrm{CO}_{2}$, and moved to the dark, and incubation was continued a further 30 minutes at room temperature. Cells were imaged live using a Leica SP5 confocal microscope fitted with a $\times 20$ dry objective. Movies were recorded at 1 frame/1.29 seconds for 60 frames before ligand addition and imaged for a further 60 minutes after ligand addition. The intensity of each responding cell was measured using the Image (NIH) plug-in Time Series Analyzer. The fluorescence intensity was obtained by subtraction of the average background intensity for each cell calculated from the average intensity across the first 60 frames (before ligand addition). Changes in fluorescence across the time course, i.e., bleaching or stress-induced fluorescence, were then accounted for across each frame by subtraction of the average background fluorescence from 30 nonresponding cells within the same field of view, allowing normalization of each cell to its own background fluorescence, and normalization of background fluorescence across all frames. 
Statistics. Data are expressed as mean \pm SEM, unless otherwise stated. Unpaired 2-tailed Student's $t$ tests and ANOVA tests were performed using GraphPad Prism 8.

\section{Effects of MVT-602 and KP54 on electrophysiological recordings in GnRH neurons}

Details of animals, brain slice preparation, electrophysiological recordings, and statistical analysis of electrophysiological studies are presented in Supplemental Methods.

Experimental design. To study the effects of MVT-602 on GnRH neurons compared with KP54, we executed extracellular recordings as a blind experiment. All drug stocks were reconstituted in water and diluted to a final concentration of $10 \mathrm{nM}$ in artificial cerebrospinal fluid for treatment. Once the extracellular recording configuration was established, recordings were stabilized for 5-10 minutes, and then spontaneous basal (control) activity was recorded for 10 minutes. Either $10 \mathrm{nM} \mathrm{KP5} 4$ or $10 \mathrm{nM}$ MVT-602 was then bath-applied for 5 minutes, followed by a wash period of at least 40 minutes to determine whether effects were reversible. No more than 2 cells per mouse were recorded with only 1 cell per brain slice. The range of firing rate observed within an animal was similar to that among animals within a group. All recorded neurons were mapped to a brain atlas (62) to determine the relation between anatomical location and response to treatment. No correlation between the location of cells and response was observed in this study.

Action currents during targeted extracellular recording reflect action potential firing. Action currents were detected using custom software written in Igor Pro (WaveMetrics). Data were binned at 5 -minute intervals, and mean firing rate (number of action potentials per 5-minute bin) was calculated for control (last 5 minutes of control period), treatment (last 3 minutes of treatment and first 2 minutes of washout period; the first 2 minutes of the treatment are skipped to allow for solution exchange), and washout periods (sequential 5-minute bins). Cells were defined as responding if firing frequency during treatment changed by $\geq 30 \%$. One cell from each group did not respond to treatment and was thus tested with first KP10 (Phoenix Pharmaceuticals) and then $20 \mathrm{mM} \mathrm{KCl}$. Neither cell responded to KP10, but both responded to $\mathrm{KCl}$, indicating technically acceptable recordings but lack of response to kisspeptin. Because these recordings were of short duration and included the additional quality tests, they were excluded from the analysis and are reported as nonresponding cells.

\section{Author contributions}

All authors provided contributions to study conception and design, acquisition of data, or analysis and interpretation of data; drafting of the article; critical revision of the article for important intellectual content; and final approval of the version to be published. Here are the most important contributions of each author: AA, PCE, MP, SAC, RR, CMS, CP, SM, AH, and WSD designed the study; AA, PCE, MP, SAC, RR, CMS, CP, EM, MM, CIE, DP, and ANC conducted experiments and acquired the data; AA, PCE, MP, SAC, DP, $\mathrm{KP}, \mathrm{CNJ}, \mathrm{LW}, \mathrm{RS}$, and ANC recruited patients for the clinical studies; AA, PCE, MP, SAC, RR, CMS, CP, BO, CAM, MV, KTA, SM, $\mathrm{AH}$, and WSD analyzed the data; AA, PCE, MP, EM, CIE, PB, and ANC conducted assays for biochemical analytes; and all authors contributed to writing and revision of the manuscript. WSD takes final responsibility for this article. The order of the co-first authors was determined by the degree of their contributions to the study.

\section{Acknowledgments}

The study was an investigator-led study (WSD) designed, conducted, analyzed, and reported entirely by the authors. This paper presents independent research funded by grants from the National Institute for Health Research (NIHR) and supported by the NIHR/Wellcome Trust Imperial Clinical Research Facility and the NIHR Imperial Biomedical Research Centre. The Section of Endocrinology and Investigative Medicine was funded by grants from the Medial Research Council (MRC), the Biotechnology and Biological Sciences Research Council (BBSRC), and the NIHR and was supported by the NIHR Biomedical Research Centre Funding Scheme. The views expressed are those of the authors and not necessarily those of the MRC, the BBSRC, the National Health Service, the NIHR, or the Department of Health. AA was supported by NIHR Clinician Scientist Award CS-2018-18-ST2-002. SAC was supported by funding from an NIHR Academic Clinical Lectureship. CNJ was supported by an NIHR Post-Doctoral Fellowship. KTA and MV gratefully acknowledge the financial support of the Engineering and Physical Sciences Research Council via grant EP/N014391/1. SM was supported by NIH/Eunice Kennedy Shriver National Institute of Child Health and Human Development grant R37 HD34860. WSD was supported by an NIHR Research Professorship (NIHR-RP-2014-05-001). We are grateful to Andy V. Babwah (Rutgers University, New Jersey) for providing the FLAG-tagged human KISS1R/pcDNA3 plasmid.

Address correspondence to: Waljit S. Dhillo, Department of Investigative Medicine, Imperial College London, 6th Floor, Commonwealth Building, Hammersmith Hospital, Du Cane Road, London W12 ONN, United Kingdom. Phone: 44.208.383.3242; Email: w.dhillo@imperial.ac.uk.
1. de Roux N, Genin E, Carel JC, Matsuda F, Chaussain JL, Milgrom E. Hypogonadotropic hypogonadism due to loss of function of the KiSS1-derived peptide receptor GPR54. Proc Natl Acad Sci U S A. 2003;100(19):10972-10976.

2. Seminara SB, et al. The GPR54 gene as a regulator of puberty. N Engl JMed.2003;349(17):1614-1627.

3. Castellano JM, et al. Changes in hypothalamic KiSS-1 system and restoration of pubertal activation of the reproductive axis by kisspeptin in undernutrition. Endocrinology. 2005;146(9):3917-3925.
4. Han SK, et al. Activation of gonadotropin-releasing hormone neurons by kisspeptin as a neuroendocrine switch for the onset of puberty. J Neurosci. 2005;25(49):11349-11356.

5. Roa J, et al. Metabolic regulation of female puberty via hypothalamic AMPK-kisspeptin signaling. Proc Natl Acad Sci U S A. 2018;115(45):E10758-E10767.

6. Smith JT, Clay CM, Caraty A, Clarke IJ. KiSS1 messenger ribonucleic acid expression in the hypothalamus of the ewe is regulated by sex steroids and season. Endocrinology. 2007;148(3):1150-1157.
7. Ohtaki T, et al. Metastasis suppressor gene KiSS-1 encodes peptide ligand of a G-protein-coupled receptor. Nature. 2001;411(6837):613-617.

8. Dhillo WS, et al. Kisspeptin-54 stimulates the hypothalamic-pituitary gonadal axis in human males. JClin Endocrinol Metab. 2005;90(12):6609-6615.

9. Dhillo WS, et al. Kisspeptin-54 stimulates gonadotropin release most potently during the preovulatory phase of the menstrual cycle in women. J Clin Endocrinol Metab. 2007;92(10):3958-3966.

10. Chan YM, Butler JP, Sidhoum VF, Pinnell NE, 
Seminara SB. Kisspeptin administration to women: a window into endogenous kisspeptin secretion and GnRH responsiveness across the menstrual cycle. JClin Endocrinol Metab. 2012;97(8):E1458-E1467.

11. Hunjan T, Abbara A. Clinical translational studies of kisspeptin and neurokinin B. Semin Reprod Med. 2019;37(3):119-124.

12. Jayasena $\mathrm{CN}$, et al. Kisspeptin-54 triggers egg maturation in women undergoing in vitro fertilization. J Clin Invest. 2014;124(8):3667-3677.

13. Abbara A, et al. Efficacy of kisspeptin-54 to trigger oocyte maturation in women at high risk of ovarian hyperstimulation syndrome (OHSS) during in vitro fertilization (IVF) therapy. J Clin Endocrinol Metab. 2015;100(9):3322-3331.

14. Jayasena $\mathrm{CN}$, et al. Increasing LH pulsatility in women with hypothalamic amenorrhoea using intravenous infusion of Kisspeptin-54. J Clin Endocrinol Metab. 2014;99(6):E953-E961.

15. Jayasena CN, et al. Subcutaneous injection of kisspeptin-54 acutely stimulates gonadotropin secretion in women with hypothalamic amenorrhea, but chronic administration causes tachyphylaxis. JClin Endocrinol Metab. 2009;94(11):4315-4323.

16. Jayasena $\mathrm{CN}$, et al. Twice-weekly administration of kisspeptin- 54 for 8 weeks stimulates release of reproductive hormones in women with hypothalamic amenorrhea. Clin Pharmacol Ther. 2010;88(6):840-847.

17. Kotani M, et al. The metastasis suppressor gene Kiss-1 encodes kisspeptins, the natural ligands of the orphan $G$ protein-coupled receptor GPR54. J Biol Chem. 2001;276(37):34631-34636.

18. George JT, et al. Kisspeptin-10 is a potent stimulator of LH and increases pulse frequency in men. JClin Endocrinol Metab. 2011;96(8):E1228-E1236.

19. Jayasena CN, et al. Direct comparison of the effects of intravenous kisspeptin-10, kisspeptin-54 and GnRH on gonadotrophin secretion in healthy men. Hum Reprod. 2015;30(8):1934-1941.

20. Nishizawa N, et al. Design and synthesis of an investigational nonapeptide KISS1 receptor (KISS1R) agonist, Ac-d-Tyr-hydroxyproline (Hyp)-Asn-Thr-Phe-azaGly-Leu-Arg(Me)-Trp$\mathrm{NH}_{2}$ (TAK-448), with highly potent testosterone-suppressive activity and excellent water solubility. J Med Chem. 2016;59(19):8804-8811.

21. Matsui H, Asami T. Effects and therapeutic potentials of kisspeptin analogs: regulation of the hypothalamic-pituitary-gonadal axis. Neuroendocrinology. 2014;99(1):49-60.

22. Orsini MJ, Klein MA, Beavers MP, Connolly PJ, Middleton SA, Mayo KH. Metastin (KiSS-1) mimetics identified from peptide structure-activity relationship-derived pharmacophores and directed small molecule database screening. $J$ Med Chem. 2007;50(3):462-471.

23. MacLean DB, Matsui H, Suri A, Neuwirth R, Colombel M. Sustained exposure to the investigational Kisspeptin analog, TAK-448, down-regulates testosterone into the castration range in healthy males and in patients with prostate cancer: results from two phase 1 studies. JClin Endocrinol Metab. 2014;99(8):E1445-E1453.

24. Teede HJ, et al. Recommendations from the international evidence-based guideline for the assessment and management of polycystic ovary syndrome. Hum Reprod. 2018;33(9):1602-1618.

25. Gordon CM, et al. Functional hypothalamic amenorrhea: an Endocrine Society clinical practice guideline. JClin Endocrinol Metab. 2017;102(5):1413-1439.

26. Thurston L, Abbara A, Dhillo WS. Investigation and management of subfertility. JClin Pathol. 2019;72(9):579-587.

27. Franssen D, Tena-Sempere M. The kisspeptin receptor: a key G-protein-coupled receptor in the control of the reproductive axis. Best Pract Res Clin Endocrinol Metab. 2018;32(2):107-123.

28. Bilban M, et al. Kisspeptin-10, a KisS-1/metastinderived decapeptide, is a physiological invasion inhibitor of primary human trophoblasts. JCell Sci. 2004;117(pt 8):1319-1328.

29. Hiden U, Bilban M, Knöfler M, Desoye G. Kisspeptins and the placenta: regulation of trophoblast invasion. Rev Endocr Metab Disord. 2007;8(1):31-39.

30. Pielecka-Fortuna J, Chu Z, Moenter SM. Kisspeptin acts directly and indirectly to increase gonadotropin-releasing hormone neuron activity and its effects are modulated by estradiol. Endocrinology. 2008;149(4):1979-1986.

31. Pielecka-Fortuna J, DeFazio RA, Moenter SM. Voltage-gated potassium currents are targets of diurnal changes in estradiol feedback regulation and kisspeptin action on gonadotropin-releasing hormone neurons in mice. Biol Reprod. 2011;85(5):987-995.

32. Irwig MS, et al. Kisspeptin activation of gonadotropin releasing hormone neurons and regulation of KiSS-1 mRNA in the male rat. Neuroendocrinology. 2004;80(4):264-272.

33. Hoff JD, Quigley ME, Yen SS. Hormonal dynamics at midcycle: a reevaluation. JClin Endocrinol Metab. 1983;57(4):792-796.

34. Abbara A, et al. A second dose of kisspeptin-54 improves oocyte maturation in women at high risk of ovarian hyperstimulation syndrome: a Phase 2 randomized controlled trial. Hum Reprod. 2017;32(9):1915-1924.

35. Clarkson J, d'Anglemont de Tassigny X, Moreno AS, Colledge WH, Herbison AE. Kisspeptin-GPR54 signaling is essential for preovulatory gonadotropin-releasing hormone neuron activation and the luteinizing hormone surge. J Neurosci. 2008;28(35):8691-8697.

36. Kinoshita M, et al. Involvement of central metastin in the regulation of preovulatory luteinizing hormone surge and estrous cyclicity in female rats. Endocrinology. 2005;146(10):4431-4436.

37. Abbara A, Clarke SA, Dhillo WS. Novel concepts for inducing final oocyte maturation in in vitro fertilization treatment. Endocr Rev. 2018;39(5):593-628.

38. Stricker R, Eberhart R, Chevailler MC, Quinn FA, Bischof P, Stricker R. Establishment of detailed reference values for luteinizing hormone, follicle stimulating hormone, estradiol, and progesterone during different phases of the menstrual cycle on the Abbott ARCHITECT analyzer. Clin Chem Lab Med. 2006;44(7):883-887.

39. Groome NP, et al. Measurement of dimeric inhibin B throughout the human menstrual cycle. JClin Endocrinol Metab. 1996;81(4):1401-1405
40. Vuong TN, Ho MT, Ha TD, Phung HT, Huynh GB, Humaidan P. Gonadotropin-releasing hormone agonist trigger in oocyte donors cotreated with a gonadotropin-releasing hormone antagonist: a dose-finding study. Fertil Steril. 2016;105(2):356-363.

41. George JT, Millar RP, Anderson RA. Hypothesis: kisspeptin mediates male hypogonadism in obesity and type 2 diabetes. Neuroendocrinology. 2010;91(4):302-307.

42. Abbara A, et al. Hypothalamic response to kisspeptin-54 and pituitary response to gonadotropin-releasing hormone are preserved in healthy older men. Neuroendocrinology. 2018;106(4):401-410.

43. Jayasena CN, Dhillo WS. Kisspeptin offers a novel therapeutic target in reproduction. Curr Opin Investig Drugs. 2009;10(4):311-318.

44. Abbara A, Ratnasabapathy R, Jayasena CN, Dhillo WS. The effects of kisspeptin on gonadotropin release in non-human mammals. Adv Exp Med Biol. 2013;784:63-87.

45. Jayasena CN, et al. Twice-daily subcutaneous injection of kisspeptin-54 does not abolish menstrual cyclicity in healthy female volunteers. J Clin Endocrinol Metab. 2013;98(11):4464-4474.

46. Min L, et al. Dynamic kisspeptin receptor trafficking modulates kisspeptin-mediated calcium signaling. Mol Endocrinol. 2014;28(1):16-27.

47. Babwah AV, et al. GnRH neuron-specific ablation of Gaq/11 results in only partial inactivation of the neuroendocrine-reproductive axis in both male and female mice: in vivo evidence for Kiss1r-coupled Gaq/11-independent GnRH secretion. J Neurosci. 2015;35(37):12903-12916.

48. Ahow M, et al. KISS1R signals independently of $\mathrm{G} \alpha \mathrm{q} / 11$ and triggers $\mathrm{LH}$ secretion via the $\beta$-arrestin pathway in the male mouse. Endocrinology. 2014;155(11):4433-4446.

49. Decourt C, et al. A synthetic kisspeptin analog that triggers ovulation and advances puberty. Sci Rep. 2016;6:26908.

50. Decourt C, et al. The kisspeptin analog C6 is a possible alternative to PMSG (pregnant mare serum gonadotropin) for triggering synchronized and fertile ovulations in the alpine goat. PLOS One. 2019;14(3): 0214424.

51. Caraty A, et al. Kisspeptin synchronizes preovulatory surges in cyclical ewes and causes ovulation in seasonally acyclic ewes. Endocrinology. 2007;148(11):5258-5267.

52. Glanowska KM, Moenter SM. Differential regulation of GnRH secretion in the preoptic area (POA) and the median eminence (ME) in male mice. Endocrinology. 2015;156(1):231-241.

53. Liu X, Lee K, Herbison AE. Kisspeptin excites gonadotropin-releasing hormone neurons through a phospholipase $\mathrm{C} /$ calcium-dependent pathway regulating multiple ion channels. Endocrinology. 2008;149(9):4605-4614.

54. Comninos AN, et al. Kisspeptin modulates sexual and emotional brain processing in humans. J Clin Invest. 2017;127(2):709-719.

55. d'Anglemont de Tassigny X, Jayasena CN, Murphy KG, Dhillo WS, Colledge WH. Mechanistic insights into the more potent effect of KP-54 compared to KP-10 in vivo. PLoS One. 2017;12(5):e0176821. 
56. Jacobi JS, Martin C, Nava G, Jeziorski MC, Clapp C, Martínez de la Escalera G. 17-Beta-estradiol directly regulates the expression of adrenergic receptors and kisspeptin/GPR54 system in GT1-7 GnRH neurons. Neuroendocrinology. 2007;86(4):260-269.

57. Moenter SM, Chu Z, Christian CA. Neurobiological mechanisms underlying oestradiol negative and positive feedback regulation of gonadotrophin-releasing hormone neurones. J Neuroendocrinol. 2009;21(4):327-333.

58. Bagis T, Gokcel A, Zeyneloglu HB, Tarim E, Kilicdag EB, Haydardedeoglu B. The effects of short-term medroxyprogesterone acetate and micronized progesterone on glucose metabolism and lipid profiles in patients with polycystic ovary syndrome: a prospective randomized study. JClin Endocrinol Metab. 2002;87(10):4536-4540.

59. Loucks AB, Thuma JR. Luteinizing hormone pulsatility is disrupted at a threshold of energy availability in regularly menstruating women. JClin Endocrinol Metab. 2003;88(1):297-311.

60. Baird DT, Thong KJ, Hall C, Cameron ST. Failure of oestrogen induced luteinizing hormone surge in women treated with mifepristone (RU 486) every day for 30 days. Hum Reprod.
1995;10(9):2270-2276.

61. Ahmed TA. Pharmacokinetics of Drugs Following IV Bolus, IV Infusion, and Oral Administration. IntechOpen. https://www.intechopen. com/books/basic-pharmacokineticconcepts-and-some-clinical-applications/ pharmacokinetics-of-drugs-following-ivbolus-iv-infusion-and-oral-administration. Published November 18, 2015. Accessed September 28, 2020.

62. Sidman RL, Angevine JB Jr, Pierce EP. Atlas of the Mouse Brain and Spinal Cord. Harvard University Press; 1971 